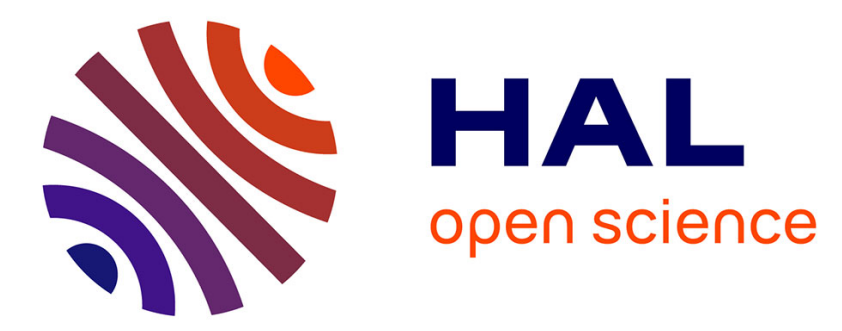

\title{
Real indeterminacy and dynamics of asset price bubbles in general equilibrium
}

\author{
Stefano Bosi, Cuong Le Van, Ngoc-Sang Pham
}

\section{To cite this version:}

Stefano Bosi, Cuong Le Van, Ngoc-Sang Pham. Real indeterminacy and dynamics of asset price bubbles in general equilibrium. 2020. halshs-02993656v1

\section{HAL Id: halshs-02993656 \\ https://shs.hal.science/halshs-02993656v1}

Preprint submitted on 6 Nov 2020 (v1), last revised 25 Sep 2021 (v2)

HAL is a multi-disciplinary open access archive for the deposit and dissemination of scientific research documents, whether they are published or not. The documents may come from teaching and research institutions in France or abroad, or from public or private research centers.
L'archive ouverte pluridisciplinaire HAL, est destinée au dépôt et à la diffusion de documents scientifiques de niveau recherche, publiés ou non, émanant des établissements d'enseignement et de recherche français ou étrangers, des laboratoires publics ou privés. 


\title{
Real indeterminacy and dynamics of asset price bubbles in general equilibrium
}

\author{
Stefano BOSI* Cuong LE VAN ${ }^{\dagger} \quad$ Ngoc-Sang PHAM $^{\ddagger}$
}

November 4, 2020

\begin{abstract}
We show that both real indeterminacy and asset price bubble may appear in an infinite-horizon exchange economy with infinitely lived agents and an imperfect financial market. We clarify how the asset structure and heterogeneity (in terms of preferences and endowments) affect the existence and the dynamics of asset price bubbles as well as the equilibrium indeterminacy. Moreover, this paper bridges the literature on bubbles in models with infinitely lived agents and that in overlapping generations models (Tirole, 1985).

Keywords: asset price bubble, real indeterminacy, borrowing constraint, intertemporal equilibrium, infinite-horizon.

JEL Classifications: D53, E44, G12.
\end{abstract}

\section{Introduction}

The existence and dynamics of asset price bubbles are one of the fundamental questions in economics and finance. According to the classical paper by Santos and Woodford (1997), conditions under which bubbles exist are relatively fragile. After the global financial crisis of 2007-2009, this topic has regained momentum and different new mechanisms of bubbles have been proposed. ${ }^{1}$ To date, the literature on rational asset price bubbles has focused on two frameworks: (1) overlapping generations models (OLG) and (2) infinite-horizon general equilibrium models with infinitely lived agents. Note that since the influential paper of Tirole (1985), numerous studies

*EPEE, Université Paris-Saclay. Email: stefano.bosi@universite-paris-saclay.fr

$\dagger$ IPAG, CNRS, PSE, and TIMAS. Email: Cuong.Le-Van@univ-paris1.fr

‡EM Normandie Business School, Métis Lab. Email: npham@em-normandie.com

${ }^{1}$ See Farhi and Tirole (2012), Martin and Ventura (2012), Gali (2014, 2018), Hirano and Yanagawa (2017), Miao and Wang (2012, 2018), Barbie and Hillebrand (2018) among others. The reader can also find excellent surveys in Brunnermeier and Oehmke (2012), Miao (2014) and Martin and Ventura (2018). 
have privileged OLG models to study the existence of bubbles and their macroeconomic implications. Although it is also important to study infinite-horizon models of bubbles, ${ }^{2}$ this type of framework has received relatively less attention. ${ }^{3}$ As recognized by Kocherlakota (2008), Miao (2014) and Martin and Ventura (2018), our understanding of bubbles in infinite-horizon models is far from complete.

The present paper aims to address basic and open questions about rational asset price bubbles in intertemporal competitive equilibrium: Why do asset price bubbles exist in equilibrium? What is the connection between the existence of bubbles on the one hand and the economic agents' consumption and trade on the other? How do the existence and dynamics of the asset price bubble depend on asset structure and economic fundamentals such as endowments?

To answer these questions, we consider an infinite-horizon general equilibrium model with a finite number of agents, where there are one consumption good and one financial asset as Lucas' tree (Lucas, 1978). Our model has two key ingredients: first, agents are heterogeneous in terms of endowments and preferences; and second, there exist financial frictions in the form of short-sale constraints, i.e., the asset quantity that each agent can buy does not exceed an exogenous limit. As in Tirole (1982), Kocherlakota (1992), Santos and Woodford (1997), given an equilibrium, we say that there exists a bubble in this equilibrium if the equilibrium asset price exceeds the fundamental value of the asset, defined as the present value of dividend streams. An equilibrium with (resp., without) bubble is said to be bubbly (resp., bubbleless).

Our contribution is three-fold.

First, we provide new conditions under which bubbles are ruled out. The literature on bubbles in infinite-horizon models shows several conditions ruling out asset price bubbles. Kocherlakota (1992) questioned the relationship between the existence of bubbles and borrowing constraints. He pointed out that, in the presence of bubbles, the limit infimum of the differences between asset holding and borrowing limit equals zero. We go further by proving that in any equilibrium with bubbles, there exist at least two agents whose borrowing constraints bind (i.e., asset holding equals borrowing limit) at infinitely many dates, and there exist at least two agents (not necessarily the same agents just mentioned) whose assets holdings fluctuate over time.

Another famous no-bubble condition in Theorem 3 in Santos and Woodford (1997), which states that, under mild conditions, bubbles are ruled out if the present value of aggregate endowments is finite. This condition still holds in a model with debt constraints (Werner, 2014) and in a model with land and collateral constraints (Bosi et al., 2018b). In our model with short-sale constraints, we also obtain this no-bubble condition (see Corollary 2).

Motivated by the fact that most of the no-bubble conditions are based on endogenous variables, we contribute to the literature by providing conditions based on

\footnotetext{
${ }^{2}$ Miao (2014) explains why we need to study infinite-horizon models of bubbles.

${ }^{3}$ In such models, it is difficult to characterize or compute the equilibrium. It is also not easy to provide non-trivial examples of equilibrium.
} 
fundamentals. The first condition (see Corollary 1) shows the role of the borrowing limits: there is no equilibrium with bubbles if borrowing limits are high enough. The second condition (see Proposition 3) shows the role of impatience: under the assumption of uniform impatience, there is no bubble if agents strongly prefer the present. The intuition is simple: if agents strongly prefer the present, they do not buy the asset in the long run, ruling out bubbles. In particular, this is the situation in finite-horizon models in which no one buys the asset in the last period eliminating the possibility of bubbles.

The second avenue of our contribution concerns the construction of models with bubbles where we can explicitly characterize the existence and the dynamics of bubbles by using fundamentals such as agents' endowments, borrowing limits, asset dividends, and asset supply.

The above no-bubble conditions suggest us to focus on a two-agent model and characterize the equilibrium in which borrowing constraints of both agents bind infinitely many dates (more precisely, the first agent's borrowing constraint will bind, for instance, at even dates and that of the second agent at odd dates). We find that such an equilibrium exists only if (1) the borrowing limits are low and (2) the benchmark economy (i.e., the economy without asset) experiences the so-called seesaw property. An economy verifies the seesaw property whenever one agent's subjective interest rate is higher than that of another agent at infinitely many dates while being lower at infinitely many other dates. Focusing on such equilibrium, we find that bubbles are ruled out if the value of endowments (discounted by using the interest rates of the benchmark economy) of the agent who buys asset vanishes in the infinity (see Proposition 4). As a consequence, there cannot exist a bubble if the benchmark interest rates are high. The basic idea is that asset buyers' income must be high enough so that they are willing to buy the asset, even when the asset price exceeds the fundamental value.

Notice that this condition concerning the benchmark interest rates is based on fundamentals and cannot be obtained from the famous condition in Santos and Woodford (1997), which is based on endogenous variables. Our finding can be viewed as an extension of the no-bubble condition of Tirole (1985) in an OLG model ${ }^{4}$ to our general equilibrium model with infinitely lived agents. In this sense, our paper is the first to create the connection between the no-bubble conditions in Tirole (1985) and those in infinite-horizon general equilibrium models. Recall that Tirole (1985), Farhi and Tirole (2012) need the convergence of interest rates of the economy without asset while we do not require such convergence.

In the existing literature, there are some examples of bubbles in general equilibrium models with infinitely lived agents. ${ }^{5}$ Concerning the asset having zero dividends

\footnotetext{
${ }^{4}$ It states that there is no bubble if the steady-state interest rate of the economy without bubble asset is higher than the population growth rate

${ }^{5}$ Brunnermeier and Oehmke (2012), Miao (2014), and Martin and Ventura (2018) provide excellent surveys on bubbles. Here, we focus on bubbles in general equilibrium models with infinitely
} 
and positive supply (i.e., fiat money), Bewley (1980) (Section 13), Townsend (1980), Kocherlakota (1992) (Example 1) and Scheinkman and Weiss (1986) show that, when borrowing is not allowed, fiat money may have positive value in infinite-horizon general equilibrium models. Santos and Woodford (1997) present several examples of this kind of bubbles: their examples 4.1, 4.2 study fiat money in deterministic models while their example 4.4 investigates fiat money in a stochastic model. Hirano and Yanagawa (2017) also give sufficient conditions for the existence of stochastic bubbles of an asset without dividend. There are a few examples of bubbles of assets with positive dividends. In a deterministic set-up, Example 4.3 in Santos and Woodford (1997) studies bubbles of an asset with positive dividends but with zero net supply. Like us, Example 4.5 in Santos and Woodford (1997) also investigates bubbles of the Lucas' tree, although they use a stochastic model with a single representative household. ${ }^{6}$ Recently, Le Van and Pham (2016), Bosi et al. (2017a), Bosi et al. (2018b) show that bubbles of assets with positive dividends and positive net supply may appear even in deterministic models. Bloise and Citanna (2019) provide a sufficient condition based on trade and punishment for default for the existence of the bubble of an asset with vanishing dividends of an equilibrium whose sequence of allocations converges.

To date, no example shows how the existence and the dynamics of asset price bubbles depend on fundamentals such as endowments and the asset structure (dividends, asset supply, and borrowing limits). Our main contribution is to fill this gap. More precisely, Section 4.3 of the present paper provides several models (without any restriction on fundamentals) where bubbles exist. Notice that studying these models is not easy because we have to work with a dynamical system that is non-stationary and has infinitely many parameters (which are our model's fundamentals). We prove that: when the benchmark economy has low interest rates and verifies the seesaw property, bubbles are more likely to exist in equilibrium if (1) asset supply is low, (2) borrowing limits of agents are low, (3) the level of heterogeneity (proxied by the differences between agents' fundamentals such as endowments, initial asset holdings, rates of time preferences) is high, and (4) asset dividends are low with respect to agents' endowments. Consequently, our results suggest that bubbles may appear if (i) the agents' endowments grow asymmetrically, and (ii) there is a shortage of financial assets (i.e., there is a low supply and assets provide low dividends). ${ }^{7}$ We also prove that bubbles may not exist if one of these four conditions is not satisfied.

The basic mechanism of asset price bubbles in our model is the following: the agents' heterogeneity and the seesaw property ensure that, at any period, at least one agent needs to save as much as possible by buying the asset. When the asset

lived agents.

${ }^{6}$ In this example, they introduce a sequence of non-stationary stochastic discount factors and show that bubbles may exist under a state-price process but not under another state-price process.

${ }^{7}$ In our model, the intertemporal utility function is time-separable. Araujo et al. (2011) consider the utility function $\sum_{t \geq 0} \zeta_{i, t} u\left(c_{i, t}\right)+\epsilon_{i} \inf _{t \geq 0} u_{i}\left(c_{i, t}\right)$ and show that the parameter $\epsilon_{i}$ plays a key role on the existence of bubbles. 
supply and borrowing limits are low, the asset price would be high (even higher than its fundamental value) because it is the only way to smooth consumption. ${ }^{8}$

To the best of our knowledge, we are the first to show that there is a continuum of bubbly equilibria in models with infinitely lived agents. With additional specifications, we can further provide a complete characterization of the set of equilibria with bubbles, and compute the bubble component as a function of fundamentals. In our models of bubbles, the asset price may converge to any value in $[0, \infty]$ or may fluctuate over time, depending on the fundamentals' properties. Furthermore, here the existence of bubbles does not violate individual transversality conditions (TVC, henceforth). Notice that individual TVC ensures the optimality of agents' choices and always holds in equilibrium while bubbles can exist or be ruled out.

Our third contribution is to clarify the relationship between the existence of bubble, equilibrium indeterminacy and (individual) welfare.

The equilibrium indeterminacy in our model is global, and no local approximation is invoked to prove this indeterminacy. Our proof relies on the fact that asset prices, in some cases, can be recursively computed. Hence the sequence of prices can be computed as a function of the initial price. Therefore, at the initial date, any value can be an equilibrium price if it is low enough so that the price and the bubble component will be not too high in the future, ensuring that agents can buy them. As a result, there may be a continuum of asset prices and a continuum of equilibrium trajectories. Notice that we require neither the convergence of these trajectories nor the existence of a steady-state. So, the indeterminacy in our model is quite different from the concept of dynamic indeterminacy in macroeconomics (see Benhabib and Farmer (1999), Farmer (2019) for surveys on this issue). Our result on indeterminacy complements the findings in Kehoe and Levine (1985), Kehoe et al. (1990) who show that in a general equilibrium model with a finite number of infinitely lived consumers and with complete financial markets, equilibria are generically determinate. ${ }^{9}$ Unlike them, we introduce financial frictions and prove that equilibria may be generically indeterminate. Moreover, the real indeterminacy in our model is associated with the existence of bubbles.

Our paper also contributes to understanding the relationship between financial assets, the existence of bubbles, and welfare. First, we prove that the equilibrium allocation in a model with bubble strictly Pareto dominates the autarkic one. The basic intuition is that the financial asset, even it contains a bubble component, provides two ways to smooth consumption: saving and borrowing. Thanks to this, agents can transfer their wealth from dates with high endowments to dates with low endowments. Second and more importantly, we show in Proposition 9 that, in our models where there are multiple equilibria, the allocation of any bubbly equilibrium strictly Pareto

\footnotetext{
${ }^{8}$ We can prove that, if we introduce a new asset with which agents can borrow without limit, there will be no bubble in equilibrium.

${ }^{9}$ More precisely, under conditions in Proposition 2 in Kehoe et al. (1990), there is a finite (odd) number of equilibria.
} 
dominates that of the bubbleless equilibrium (notice that the bubbleless equilibrium is not necessarily the autarkic one). The idea is that both the asset prices and the bubble component increase with the initial price. Consequently, the initial asset price of the bubbleless equilibrium is lower than that of any bubbly equilibrium. When the price increases, it helps to reduce the marginal rate of substitution of agents, which in turn allows agents to smooth their consumption. Therefore, individual welfare generated by any bubbly equilibrium is higher than that generated by the bubbleless one. This point is consistent with Proposition 9 of Hirano and Yanagawa (2017). The difference is that we work under general utility functions while they only focus on the logarithmic utility function.

The rest of the paper is organized as follows. Section 2 presents the framework and provides the fundamental properties of equilibrium. Sections 3 provides no-bubble conditions. A number of models with bubbles and real indeterminacy are presented in Section 4. Finally, Section 5 concludes and mentions future works. Technical proofs are gathered in the appendices.

\section{An exchange economy with short-sale constraints}

Consider an infinite-horizon discrete-time model with short-sale as in Kocherlakota (1992). There are a finite number $m$ of agents, a single consumption good and an asset. The asset structure is similar to Lucas' tree (Lucas, 1978) with exogenous dividend stream $\left(d_{t}\right)_{t}$. Denote $c_{i, t}, b_{i, t}$ the consumption and asset holding of agent $i$ at date $t$ while $q_{t}$ is the asset price at date $t$. Agent $i$ maximizes her intertemporal utility $\sum_{t=0}^{+\infty} \beta_{i, t} u_{i}\left(c_{i, t}\right)$ subject to the following constraints:

(1) Physical constraints: $c_{i, t} \geq 0 \forall t, \forall i$.

(2) Budget constraint: $c_{i, t}+q_{t} b_{i, t} \leq e_{i, t}+\left(q_{t}+d_{t}\right) b_{i, t-1} \forall t, \forall i$, where $e_{i, t}>0$ is the exogenous endowment of agent $i$ at date $t$ and $b_{i,-1}$ is endogenously given.

(3) Borrowing constraint (or short-sale constraint): $b_{i, t} \geq-b_{i}^{*} \forall t, \forall i$ where $b_{i}^{*} \geq 0$ is an exogenous borrowing limit.

An equilibrium is a list of prices and allocations $\left(q_{t},\left(c_{i, t}, b_{i, t}\right)_{i}\right)_{t \geq 0}$ satisfying three conditions: (1) given price, for any $i$, the allocation $\left(c_{i, t}, b_{i, t}\right)_{i}$ is a solution of the optimization problem of agent $i$ (i.e., $\sum_{t=0}^{+\infty} \beta_{i, t} u_{i}\left(c_{i, t}\right) \geq \lim \sup _{T \rightarrow \infty} \sum_{t=0}^{T} \beta_{i, t} u_{i}\left(c_{i, t}^{\prime}\right)$ for any sequence $\left(c_{i}^{\prime}, b_{i}^{\prime}\right)$ satisfying physical, budget and borrowing constraints), and (2) market clearing conditions: $\sum_{i} b_{i, t}=L$ and $\sum_{i} c_{i, t}=\sum_{i} e_{i, t}+L d_{t} \forall t \geq 0$, and (3) $q_{t}>0 \forall t$.

Denote $W_{t} \equiv \sum_{i} e_{i, t}+L d_{t}$ the aggregate resource at date $t$. We require standard assumptions in the rest of the paper.

Assumption 1. Assume that $u_{i}$ is concave, strictly increasing, and continuously differentiable for any $i$. We also assume that $\beta_{i, t}>0, e_{i, t}>0, b_{i,-1} \geq-b_{i}^{*}, d_{t} \geq 0$, $\sum_{t} \beta_{i, t} u_{i}\left(W_{t}\right)<\infty \forall i, t$, and the net asset supply is positive $(L>0)$. 
Assumption 2. There exists an increasing function $v(c)$ such that $u_{i}^{\prime}(c) c \leq v(c) \forall c$ and $\sum_{t} \beta_{i, t} v\left(W_{t}\right)<\infty \forall i$.

Notice that when $\sum_{t} \beta_{i, t}<\infty \forall i$, and $u_{i}(c)=\ln (c) \forall c, \forall i$ or $u_{i}(0)$ is finite for any $i$, Assumption 2 is a direct consequence of Assumption 1.

We start our exposition with the following result which plays a fundamental role in understanding bubbles.

Proposition 1. Let Assumption 1 be satisfied.

(1) If $\left(q,\left(c_{i}, b_{i}\right)_{i}\right)$ is an equilibrium, we have first-order conditions (FOC):

$$
\begin{aligned}
& \beta_{i, t} u_{i}^{\prime}\left(c_{i, t}\right)=\lambda_{i, t} \\
& \lambda_{i, t} q_{t}=\lambda_{i, t+1}\left(q_{t+1}+d_{t+1}\right)+\eta_{i, t}, \quad \eta_{i, t}\left(b_{i, t}+b_{i}^{*}\right)=0, \quad \eta_{i, t} \geq 0 .
\end{aligned}
$$

for any $i, t$. In addition, if Assumption 2 holds, then $\lim _{t \rightarrow \infty} \lambda_{i, t} q_{t}\left(b_{i, t}+b_{i}^{*}\right)=0$.

(2) If the sequences $\left(q,\left(c_{i}, b_{i}\right)_{i}\right)$ and $\left(\lambda_{i}, \eta_{i}\right)$ satisfy

(a) $c_{i, t}, b_{i, t}, \lambda_{i, t}, \eta_{i, t} \geq 0, q_{t} \geq 0, b_{i, t} \geq-b_{i}^{*}, c_{i, t}+q_{t} b_{i, t}=e_{i, t}+\left(q_{t}+d_{t}\right) b_{i, t-1} \forall i, t$;

(b) First-order conditions (1a-1b), and market clearing conditions;

(c) Transversality conditions (TVC): $\lim _{t \rightarrow \infty} \lambda_{i, t} q_{t}\left(b_{i, t}+b_{i}^{*}\right)=0 \forall i$;

(d) For any $i$, the series $\sum_{t=0}^{\infty} \beta_{i, t} u_{i}\left(c_{i, t}\right)$ converges.

then $\left(q,\left(c_{i}, b_{i}\right)_{i}\right)$ is an equilibrium.

Proof. See Appendix A.

Proposition 1 provides necessary and sufficient conditions under which a list of prices and allocation constitutes an equilibrium. ${ }^{10}$ Kocherlakota (1992) considers a particular function $\sum_{t} \beta_{i}^{t} u_{i}\left(c_{i, t}\right)$ and states a similar result but he requires that $u_{i}(c) \leq 0 \forall c$ or $u_{i}(c) \geq 0 \forall c$ (to ensure that the sum $\sum_{t} \beta_{i}^{t} u_{i}\left(c_{i, t}\right)$ always converges). Of course, his condition is not satisfied if $u_{i}(c)=\ln (c)$. Our result is more general and also applies to unbounded utility functions, including $u_{i}(c)=\ln (c)$. Our result is related to Proposition 1 in Bosi et al. (2018b). The difference is that we impose exogenous borrowing limits while Bosi et al. (2018b) consider collateral constraints and the borrowing limits depends on prices of assets in the future.

Our proof of TVC is quite different from that of Kamihigashi (2002). We cannot directly apply the result in Kamihigashi (2002) because he only considers positive allocations while $b_{i, t}$ may be negative in our model. It is interesting to notice that when $u_{i}(0) \geq 0 \forall i$, the second statement of Proposition 1 still holds if we replace $\lim _{t \rightarrow \infty} \lambda_{i, t} q_{t}\left(b_{i, t}+b_{i}^{*}\right)=0 \forall i$ by $\liminf _{t \rightarrow \infty} \lambda_{i, t} q_{t}\left(b_{i, t}+b_{i}^{*}\right)=0 \forall i{ }^{11}$

Following the standard literature (Tirole, 1982, 1985; Kocherlakota, 1992; Santos and Woodford, 1997), we introduce the notion of rational asset price bubbles.

\footnotetext{
${ }^{10}$ For the existence of equilibrium, see, among others, Bosi et al. (2018b) and references therein.

${ }^{11}$ See Remark 8 in Appendix A for a proof.
} 
Definition 1. Consider an equilibrium. We define discount factors $\left(R_{t}\right)_{t}$ by $R_{t+1} q_{t}=$ $q_{t+1}+d_{t+1}$. The fundamental value of the asset is $F V_{0} \equiv \sum_{t=1}^{\infty} Q_{t} d_{t}$ where $Q_{t} \equiv$ $\frac{1}{R_{1} \cdots R_{t}}$. We say that there is a bubble in this equilibrium if $q_{0}>F V_{0}$. In this case, this equilibrium is called bubbly. Otherwise, it is called bubbleless.

Remark 1. One can prove that $1=R_{t+1} \max _{i} \frac{\beta_{i, t+1} u_{i}^{\prime}\left(c_{i, t+1}\right)}{\beta_{i, t} u_{i}^{(}\left(c_{i, t}\right)} \forall t \geq 0 .{ }^{12}$

In our deterministic framework, the sequence of discount factors $\left(R_{t}\right)$ is uniquely determined. The reader is referred to Santos and Woodford (1997), Araujo et al. (2011), Pascoa et al. (2011), Bosi et al. (2018b) among others for the notion of bubbles in stochastic economies where discount factors (and state price processes) are not necessarily uniquely determined..$^{13}$

According to the asset pricing equation $q_{t}=\left(q_{t+1}+d_{t+1}\right) / R_{t+1}$, we have

$$
q_{0}=\sum_{s=1}^{t} Q_{s} d_{s}+Q_{t} q_{t} \forall t \geq 1
$$

So, there is a bubble iff $\lim _{t \rightarrow} Q_{t} q_{t}>0$, i.e., the discounted value of 1 unit of the asset does not vanish in the infinity. In a particular case where $d_{t}=0 \forall t$, the fundamental value equals zero; in this case, there is a bubble iff the asset price is strictly positive (this is the notion of bubble in Tirole (1985)).

Our main goal is to understand conditions under which rational asset price bubbles may exist (or be ruled out) in equilibrium as well as the implications of this phenomenon.

\section{No-bubble conditions}

This section aims to study necessary conditions for the existence of bubbles and find out new conditions under which bubbles cannot appear.

\subsection{The role of borrowing constraints}

The relationship between the existence of bubble and borrowing constraints is questioned by Kocherlakota (1992). However, he did not investigate whether borrowing constraints are binding or not in equilibrium with bubbles. The following result explores such a relationship and shows our contribution with respect to Kocherlakota (1992) as well as the connection between the existence of bubble and the trading on the asset market.

\footnotetext{
${ }^{12}$ Indeed, let $t \geq 0$ arbitrary, then FOCs imply that $q_{t} \geq\left(q_{t+1}+d_{t+1}\right) \max _{i} \frac{\beta_{i, t+1} u_{i}^{\prime}\left(c_{i, t+1}\right)}{\beta_{i, t} u_{i}^{\prime}\left(c_{i, t}\right)}$. Since $\sum_{i} b_{i, t}=L>0$, there is an agent $i_{t}$ such that $b_{i_{t}, t}>0$. Hence, $\eta_{i, t}=0$. By consequence, $q_{t}=\left(q_{t+1}+d_{t+1}\right) \frac{\beta_{i_{t}, t+1} u_{i_{t}}^{\prime}\left(c_{i_{t}, t+1}\right)}{\beta_{i_{t}, t} u_{i_{t}}^{t}\left(c_{i_{t}, t}\right)}$. Therefore, we obtain our result.

${ }^{13}$ See Miao and Wang $(2012,2018)$ for the notion of bubble on the value of firm and Becker et al. (2015), Bosi et al. (2017a) for the notion of bubble on physical capital.
} 
Proposition 2 (bubble existence and borrowing constraint). Let Assumption 1, 2 be satisfied. If there is a bubble in equilibrium, then we have:

1. (Kocherlakota, 1992) $\liminf _{t \rightarrow \infty}\left(b_{i, t}+b_{i}^{*}\right)=0 \forall i$.

2. There exist at least 2 agents whose borrowing constraints bind infinitely often. Formally, there exist at least 2 agents, say $i, j$, and 2 infinite increase sequences $\left(i_{n}\right)_{n},\left(j_{n}\right)_{n}$ such that $b_{i, i_{n}}+b_{i}^{*}=0$ and $b_{j, j_{n}}+b_{j}^{*}=0$ for all $n$.

3. There exist at least 2 agents $i$ and $j$ such that the sequences $\left(b_{i, t}\right)_{t}$ and $\left(b_{j, t}\right)_{t}$ do not converge.

Proof. See Appendix A.

Points 2 and 3, which complement the result in Kocherlakota (1992), show that the existence of bubbles implies the fluctuations of asset trading of at least 2 agents. Let us provide a sketch and intuition of our proof. When the borrowing constraint of any agent $i$ is not binding from some date, say $t_{0}$, then $\eta_{i, t}=0 \forall t \geq t_{0}, \forall i$, and hence $\frac{\lambda_{i, t+1}}{\lambda_{i, t}}=\frac{q_{t}}{q_{t+1}+d_{t+1}}=\frac{1}{R_{t+1}}$ and $Q_{t}=\lambda_{i, t} \frac{Q_{t_{0}}}{\lambda_{i, t_{0}}} \forall t \geq t_{0}, \forall i$. It means that the discount factor of any agent is proportional to that of the economy. So, the TVC which ensures the optimality of agent $i$ 's allocation implies that $\lim _{t \rightarrow \infty} Q_{t} q_{t}\left(b_{i, t}+b_{i}^{*}\right)=0$. When bubbles exist (i.e., $\lim _{t \rightarrow \infty} Q_{t} q_{t}>0$ ), this equality cannot hold for all $i$ because the asset is in positive net supply $\sum_{i} b_{i, t}=L>0 \forall t$.

Proposition 2's point 2 leads to the following result showing the role of borrowing limits $\left(b_{i}^{*}\right)$ on the existence of bubbles.

Corollary 1. Let Assumption 1, 2 be satisfied. If there is a date $T$ such that $b_{i}^{*} d_{t}>e_{i, t}$ $\forall i, \forall t \geq T$, then there is no equilibrium with bubble. ${ }^{14}$

Notice that this result still applies for the case where borrowing limits are not stationary, i.e., when the borrowing constraint of agent $i$ at date $t$ is $b_{i, t}+b_{i, t}^{*} \geq 0$ where $b_{i, t}^{*} \geq 0$ is exogenous.

\subsection{Interest rates, impatience and bubble}

A famous result in Santos and Woodford (1997) states that, under the assumption of uniform impatience (see infra), bubbles are ruled out if the present value of total future resources is finite (this condition was named "high implied interest rates" by Alvarez and Jermann (2000)). ${ }^{15}$ In our model with short-sale constraints, we can also prove a similar result.

\footnotetext{
${ }^{14}$ Indeed, suppose that there is an equilibrium with bubble. According to point 2 of Proposition 2 , there is an agent $i$ and an infinite sequence $\left(i_{n}\right)_{n}$ such that $b_{i, i_{n}}+b_{i}^{*}=0 \forall n$. Let $n$ be such that $i_{n}>T$. We have $c_{i, i_{n}+1}=e_{i, i_{n}+1}-d_{i_{n}+1} b_{i}^{*}-q_{i_{n}+1}\left(b_{i}^{*}+b_{i, i_{n}}\right) \leq e_{i, i_{n}+1}-d_{i_{n}+1} b_{i}^{*}<0$, a contradiction.

${ }^{15}$ Theorem 6.1 in Huang and Werner (2000) provides a version of Santos and Woodford (1997)'s Theorem 3 in a model with debt constraints. Proposition 12 in Bosi et al. (2018b) shows a related result concerning the bubbles of land.
} 
Corollary 2. Let Assumption 1, 2 be satisfied. There is no bubble if

$$
\sum_{t \geq 0} Q_{t}\left(\sum_{i} e_{i, t}\right)<\infty
$$

Consequently, there is no bubble if $\lim \inf _{t \rightarrow \infty} \frac{d_{t}}{\sum_{i} e_{i, t}}>0$.

Proof. See Appendix A.

Although Santos and Woodford (1997) work in a stochastic model with multiple assets, their main results require the uniform impatience. By contrast, we do not require the uniform impatience. Instead, we use transversality conditions in Proposition 1. We provide here a sketch of proof. Condition (3) is used to prove that, for any agent $i$, the discounted value of asset holding $Q_{t} q_{t} b_{i, t}$ converges when $t$ tends to infinity. If there is a bubble (i.e., $\lim _{t \rightarrow \infty} Q_{t} q_{t}>0$ ), then the sequence $\left(b_{i, t}\right)$ converges for any $i$. By market clearing condition, there is at least one agent, say $j$, whose asset holding $b_{j, t}$ converges to a strictly positive value. So, this agent's borrowing constraint is not binding from some date on. By consequence, the TVC implies that $\lim _{t \rightarrow \infty} Q_{t} q_{t}\left(b_{j, t}+b_{j}^{*}\right)=0$ which is a contradiction.

Corollary 2 also indicates bubbles can exist only if there is an infinite sub-sequence of times $\left(t_{n}\right)_{n \geq 0}$ such that the ratio $\frac{d_{t_{n}}}{\sum_{i} e_{i, t_{n}}}$ converges to zero, i.e., the dividend will be very low with respect to the aggregate endowment. This condition is consistent with those in Le Van and Pham (2016), Bosi et al. (2018b).

Our goal in this subsection is to find out other conditions (based on fundamentals) under which bubbles cannot appear. To do so, we borrow the concept "uniform impatience" in the existing literature (Magill and Quinzii, 1994, 1996; Levine and Zame, 1996). Given a consumption plan $c=\left(c_{t}\right)_{t \geq 0}$, a date $t$, a vector $(\gamma, \delta) \in$ $(0,1) \times \mathbb{R}_{+}$, we define another consumption plan, called $z=z(c, t, \gamma, \delta)$, by $z_{s}=$ $c_{s} \forall s<t, z_{t}=c_{t}+\delta, z_{s}=\gamma c_{s} \forall s>t$. We also denote $U_{i}^{T}(c)=\sum_{t=0}^{T} \beta_{i, t} u_{i}\left(c_{i, t}\right)$ and $U_{i}(c) \equiv \lim \sup _{T \rightarrow \infty} U_{i}^{T}(c)$.

Assumption 3 (Uniform impatience). There exists $\gamma \in(0,1)$ such that for any consumption plan $c=\left(c_{t}\right)$ with $0 \leq c_{t} \leq W_{t} \forall t$, we have

$$
U_{i}\left(z\left(c, t, \gamma^{\prime}, W_{t}\right)\right)>U_{i}(c) \forall i, \forall t, \forall \gamma^{\prime} \in[\gamma, 1) .
$$

Proposition 1 in Pascoa et al. (2011) provides sufficient conditions for the uniform impatience. Notice that they only consider the case where $u_{i}(c) \geq 0 \forall c, \forall i$. If $u_{i}(c)=$ $\frac{c^{1-\sigma}}{1-\sigma} \forall i$, where $\sigma>0$, and there exists $\gamma \in(0,1)$ such that $\beta_{i, t} \frac{2^{1-\sigma}-1}{1-\sigma} W_{t}^{1-\sigma}+\left(\gamma^{1-\sigma}-\right.$ 1) $\sum_{s=t+1}^{\infty} \beta_{i, s} \frac{W_{s}^{1-\sigma}}{1-\sigma}>0 \forall t$, then the uniform impatience holds. We can also consider logarithmic utility functions: If $u_{i}(c)=\ln (c) \forall c, \forall i$ and there exists $\gamma \in(0,1)$ such that $\beta_{i, t}>-\frac{\ln (\gamma)}{\ln (2)} \sum_{s=t+1}^{\infty} \beta_{i, s} \forall t$, then the uniform impatience holds. ${ }^{16}$

\footnotetext{
${ }^{16}$ For a proof, see Lemma 1 in our working paper version Bosi et al. (2019).
} 
Our main contribution in this subsection can be stated as follows.

Proposition 3. Assume that Assumptions 1, 2, 3 hold and $e_{i, t}-d_{t} b_{i}^{*}>0 \forall i, \forall t$. There is no bubble if

$$
\lim _{T \rightarrow \infty} W_{T} \prod_{t=0}^{T-1} \max _{i} \frac{\beta_{i, t+1} u_{i}^{\prime}\left(e_{i, t+1}-d_{t+1} b_{i}^{*}\right)}{\beta_{i, t} u_{i}^{\prime}\left(\sum_{i} e_{i, t}+L d_{t}\right)}=0 .
$$

This leads to two consequences.

1. When $u_{i}(c)=\ln (c), \beta_{i, t}=\beta^{t} \forall i, \forall t$, and $\frac{1-\beta}{\beta}>-\frac{\ln (\gamma)}{\ln (2)}$ with $\gamma \in(0,1)$, there is no bubble if

$$
\lim _{T \rightarrow \infty} \beta^{T} W_{T} \cdots W_{1} W_{0} \prod_{t=0}^{T-1} \max _{i} \frac{1}{e_{i, t+1}-d_{t+1} b_{i}^{*}}=0 .
$$

2. When $u_{i}(c)=\frac{c^{1-\sigma}}{1-\sigma}$ where $\sigma>0$, and there exists $\gamma \in(0,1)$ such that $\frac{2^{1-\sigma}-1}{1-\sigma} W_{t}^{1-\sigma}+$ $\left(\gamma^{1-\sigma}-1\right) \sum_{s=t+1}^{\infty} \beta^{s-t} \frac{W_{s}^{1-\sigma}}{1-\sigma}>0 \forall t$, there is no bubble if

$$
\lim _{T \rightarrow \infty} \beta^{T} W_{T} \prod_{t=0}^{T-1} \max _{i} \frac{W_{t}^{\sigma}}{\left(e_{i, t+1}-d_{t+1} b_{i}^{*}\right)^{\sigma}}=0 .
$$

Proof. See Appendix A.

Proposition 3 and Corollary 1 contribute to the literature by providing conditions (based on fundamentals) under which bubbles are ruled out. The basic intuition is that the value of bubble $\lim _{t \rightarrow} \frac{q_{t}}{R_{1} \cdots R_{t}}$ must be zero if the discount factors $\left(R_{t}\right)_{t}$ are high enough. There are two key points helping us to get (4). First, we use the uniform impatience to find an upper bound of asset price $q_{t}: q_{t} \leq \frac{m W_{t}}{L(1-\gamma)} \forall t$. Second, and more importantly, by deriving an upper bound on the intertemporal marginal rate of substitution of asset holders, we can find an upper bound of $1 / R_{t}$ :

$$
\frac{1}{R_{t+1}} \leq \max _{i} \frac{\beta_{i, t+1} u_{i}^{\prime}\left(e_{i, t+1}-d_{t+1} b_{i}^{*}\right)}{\beta_{i, t} u_{i}^{\prime}\left(\sum_{i} e_{i, t}+L d_{t}\right)} \forall t \geq 0 .
$$

and so an upper bound of the discount factor $Q_{t}$. By consequence, we obtain (4).

When borrowing limits $\left(b_{i}^{*}\right)$ and dividends $\left(d_{t}\right)$ are high (in the sense that $e_{i, t}<d_{t} b_{i}^{*}$ $\forall i, \forall t)$, Corollary 1 shows that bubbles do not exist. When borrowing limits $\left(b_{i}^{*}\right)$ are low (in the sense that $e_{i, t}>d_{t} b_{i}^{*} \forall i, \forall t$ ), Proposition 3 indicates that bubbles do not exist if the agents prefer strongly the present (formally, $\beta_{i, t+1} / \beta_{i, t}$ is low). In a particular case, where $\beta_{i, t}=\beta^{t}$ with $\beta$ is low enough, there is no bubble. Notice that, when there is $T$ such that $\beta_{i, t}=0 \forall i, \forall t>T$, we recover a T-horizon model where we have $q_{0}=\sum_{s=1}^{T} Q_{s} d_{s}$ and $q_{s}=0 \forall s>T$, and therefore, there is no bubble.

In the case of zero dividends $\left(d_{t}=0 \forall t\right)$, conditions (4-6) do not depend on borrowing limits $b_{i}^{*}$. So, bubbles may be ruled out even borrowing limits are too low. This in turn suggests that financial frictions are only necessary conditions for asset price bubbles. 


\section{Models with bubbles}

We are now interested in constructing model economies in which bubbles exist. Proposition 2 shows that such models must contain at least 2 heterogeneous agents. So, we should focus on a model with two types of agents, say 1 and $2 .{ }^{17}$ Suggesting by points 2 and 3 of Proposition 2, we should look at equilibria in which borrowing constraints of agent 1 (agent 2) bind at any even (odd) date because this is the simplest model under which bubbles may exist. Formally, we aim to find economies where there is an equilibrium such that

$$
b_{1,2 t}=-b_{1}^{*}, \quad b_{2,2 t}=L+b_{1}^{*}, \quad b_{1,2 t+1}=L+b_{2}^{*}, \quad b_{2,2 t+1}=-b_{2}^{*} .
$$

With these asset holdings, we have that

$$
\begin{aligned}
c_{1,0} & =e_{1,0}+\left(q_{0}+d_{0}\right) b_{1,-1}+q_{0} b_{1}^{*}, & c_{2,0}=e_{2,0}+\left(q_{0}+d_{0}\right) b_{2,-1}-q_{0}\left(L+b_{1}^{*}\right) \\
c_{1,2 t-1} & =e_{1,2 t-1}-b_{1}^{*} d_{2 t-1}-q_{2 t-1} H, & c_{2,2 t-1}=e_{2,2 t-1}+d_{2 t-1}\left(L+b_{1}^{*}\right)+q_{2 t-1} H \\
c_{1,2 t} & =e_{1,2 t}+d_{2 t}\left(L+b_{2}^{*}\right)+q_{2 t} H, & c_{2,2 t}=e_{2,2 t}-d_{2 t} b_{2}^{*}-q_{2 t} H
\end{aligned}
$$

where $H \equiv L+b_{1}^{*}+b_{2}^{*}$ and $b_{1,-1}, b_{2,-1}$ are given. Observe that such equilibrium exists only if $e_{1,2 t-1}-b_{1}^{*} d_{2 t-1}>0$ and $e_{2,2 t}-d_{2 t} b_{2}^{*}>0 \forall t$ (we can interpret that the borrowing limits $b_{1}^{*}, b_{2}^{*}$ are low).

Denote $\gamma_{1, t} \equiv \frac{\beta_{1, t+1}}{\beta_{1, t}}, \gamma_{2, t} \equiv \frac{\beta_{2, t+1}}{\beta_{2, t}} \forall t \geq 0$. According to FOCs in Proposition 1, we have that, for any $t \geq 1$,

$$
\left\{\begin{array}{l}
1=\gamma_{1,2 t-1} \frac{u_{1}^{\prime}\left(e_{1,2 t}+d_{2 t}\left(L+b_{2}^{*}\right)+q_{2 t} H\right)}{u_{1}^{\prime}\left(e_{1,2 t-1}-b_{1}^{*} d_{2 t-1}-q_{2 t-1} H\right)} \frac{q_{2 t}+d_{2 t}}{q_{2 t-1}} \\
1=\gamma_{2,2 t} \frac{u_{2}^{\prime}\left(e_{2,2 t+1}+d_{2 t+1}\left(L+b_{1}^{*}\right)+q_{2 t+1} H\right)}{u_{2}^{\prime}\left(e_{2,2 t}-b_{2}^{*} d_{2 t}-q_{2 t} H\right)} \frac{q_{2 t+1}+d_{2 t+1}}{q_{2 t}}
\end{array}\right.
$$

and

$$
\left\{\begin{array}{l}
\gamma_{1,2 t-1} \frac{u_{1}^{\prime}\left(e_{1,2 t}+d_{2 t}\left(L+b_{2}^{*}\right)+q_{2 t} H\right)}{u_{1}^{\prime}\left(e_{1,2 t-1}-b_{1}^{*} d_{2 t-1}-q_{2 t-1} H\right)} \geq \gamma_{2,2 t-1} \frac{u_{2}^{\prime}\left(e_{2,2 t}-d_{2 t} b_{2}^{*}-q_{2 t} H\right)}{u_{2}^{\prime}\left(e_{2,2 t-1}+d_{2 t-1}\left(L+b_{1}^{*}\right)+q_{2 t-1} H\right)} \\
\gamma_{2,2 t} \frac{u_{2}^{\prime}\left(e_{2,2 t+1}+d_{2 t+1}\left(L+b_{1}^{*}\right)+q_{2 t+1} H\right)}{u_{2}^{\prime}\left(e_{2,2 t}-d_{2 t} b_{2}^{*}-q_{2 t} H\right)} \geq \gamma_{1,2 t} \frac{u_{1}^{\prime}\left(e_{1,2 t+1}-b_{1}^{*} d_{2 t+1}-q_{2 t+1} H\right)}{u_{1}^{\prime}\left(e_{1,2 t}+d_{2 t}\left(L+b_{2}^{*}\right)+q_{2 t} H\right)}
\end{array}\right.
$$

${ }^{17}$ Our results below can be extended to a model with $n$ types of agents and asset allocation is given by

$$
\begin{aligned}
& b_{1, n t}=-b_{1}^{*}, \quad b_{1, n t+1}=-b_{1}^{*}, \ldots \quad b_{1, n t+n-1}=H_{n}-b_{1}^{*} \\
& b_{2, n t}=H_{n}-b_{2}^{*}, \quad b_{2, n t+1}=-b_{2}^{*}, \ldots \quad b_{2, n t+n-1}=-b_{2}^{*} \\
& b_{n, n t}=-b_{n}^{*}, \ldots \quad b_{n, n t+n-2}=H_{n}-b_{n}^{*}, \quad b_{n, n t+n-1}=-b_{n}^{*}
\end{aligned}
$$

where $H_{n} \equiv L+\sum_{i=1}^{n} b_{i}^{*}$. 


\subsection{The role of the benchmark economy}

In this subsection, we find out new necessary conditions (based on fundamentals) of the existence of bubbles. Our key idea is to look at the benchmark economy, i.e., the economy without asset. In such economy, we have $c_{i, t}=e_{i, t} \forall i, t$.

We now define the exogenous sequences $\left(R_{1, t}^{*}\right),\left(R_{2, t}^{*}\right),\left(R_{t}^{*}\right)$ by

$$
1=\frac{\gamma_{1, t-1} u_{1}^{\prime}\left(e_{1, t}\right)}{u_{1}^{\prime}\left(e_{1, t-1}\right)} R_{1, t}^{*}, \quad 1=\frac{\gamma_{2, t-1} u_{2}^{\prime}\left(e_{2, t}\right)}{u_{2}^{\prime}\left(e_{2, t-1}\right)} R_{2, t}^{*}, \quad \text { and } R_{t}^{*} \equiv \min \left(R_{1, t}^{*}, R_{2, t}^{*}\right)
$$

We interpret $R_{1, t}^{*}$ (resp., $R_{2, t}^{*}$ ) as the subjective real interest rate of agent 1 (resp., 2) and $R_{t}^{*}$ as the real interest rate between dates $t-1$ and $t$ in the benchmark economy.

Since the dividends and asset prices are non-negative, the FOCs imply that

$$
R_{2 t}^{*}=R_{1,2 t}^{*} \leq R_{2,2 t}^{*}, R_{2 t+1}^{*}=R_{2,2 t+1}^{*} \leq R_{1,2 t+1}^{*} \forall t \geq 1 \text {. }
$$

This condition can be interpreted as follows: the benchmark economy has a so-called seesaw property.

We also see that $R_{t+1} \equiv \frac{q_{t+1}+d_{t+1}}{q_{t}} \geq R_{t+1}^{*} \forall t \geq 2$ meaning that the interest rate of the benchmark economy is lower than that of our economy with asset. The value of asset price bubble is $b_{0}=q_{0}-F V_{0}=\lim _{t \rightarrow \infty} \frac{q_{t}}{R_{1} \cdots R_{t}}$. Since the function $u_{i}^{\prime}$ is decreasing, we have

$$
\frac{q_{t}}{R_{1} \cdots R_{t}} \leq \frac{q_{t}}{R_{1}^{*} \cdots R_{t}^{*}} \frac{u_{2}^{\prime}\left(c_{2,1}\right)}{u_{2}^{\prime}\left(c_{2,0}\right)} \frac{u_{2}^{\prime}\left(e_{2,0}\right)}{u_{2}^{\prime}\left(e_{2,1}\right)} \forall t \geq 2
$$

The positivity of the consumptions implies that $H q_{t} \leq e_{t}$, where we denote $e_{2 t} \equiv e_{2,2 t}$ and $e_{2 t+1} \equiv e_{1,2 t+1}$. So, there is no bubble if $\lim _{t \rightarrow \infty} \frac{e_{t}}{R_{1}^{*} \cdots R_{t}^{*}}=0$.

By summing up the above arguments, we obtain the following result showing the role of interest rates of the economy without asset.

Proposition 4 (the role of interest rates of the benchmark economy). Consider a model with two agents. Assume that the sequence $\left(q_{t}\right)$, asset holdings are given by (8) and agents' consumptions given by (9a-9c) constitute an equilibrium. We have

$$
\begin{aligned}
& R_{t} \geq R_{t}^{*} \forall t \geq 2 \\
& R_{2,2 t}^{*} \geq R_{1,2 t}^{*}=R_{2 t}^{*}, \quad R_{1,2 t+1}^{*} \geq R_{2,2 t+1}^{*}=R_{2 t+1}^{*} \forall t \geq 1 \quad \text { (seesaw property). }
\end{aligned}
$$

Moreover, there is no bubble if

$$
\lim _{t \rightarrow \infty} \frac{e_{t}}{R_{1}^{*} \cdots R_{t}^{*}}=0
$$

In a particular case where $e_{t}=e$ and $R_{t}^{*}=R^{*} \forall t$, there is no bubble if $R^{*}>1$. 
The term $\frac{e_{t}}{R_{1}^{*} \cdots R_{t}^{*}}$ represents the value (discounted by using the interest rates of the benchmark economy) of endowment of the agent who buys asset in the economy with asset. Proposition 4 implies that, if there is bubble, the sequence of these discounted values either diverges or converges to a strictly positive value. In the case of convergence, the existence of bubble requires that $\lim _{t \rightarrow \infty} \frac{e_{t}}{R_{1}^{*} \cdots R_{t}^{*}}>0$. The basic idea behind is that the income of asset buyers must be high enough so that these agents are willing to buy the asset even the asset price exceeds its fundamental value.

Condition (14) is new with respect to the literature of rational bubbles in infinitehorizon general equilibrium models. Notice that it is not implied by the well-known no-bubble condition $\sum_{t} Q_{t}\left(\sum_{i} e_{i, t}\right)<\infty$ (see Santos and Woodford (1997), Werner (2014), Bosi et al. (2018b)) because $R_{t} \geq R_{t}^{*} \forall t$. The novelty of condition (14) is to show the importance of interest rates of the economy without asset (these interest rates are exogenous) on the existence of bubbles in the economy with assets.

Condition (14) allows us to establish the connection between the literature of bubbles in OLG models and that in infinite-horizon models. Indeed, let us compare it with the main result in the influential paper of Tirole (1985) who studies a pure bubble asset (i.e., asset pays no dividend) in an OLG model. He provides a no-bubble condition based on fundamentals: there is no bubble if the steady state interest rates of the economy without bubble asset is higher than the population growth rate. Condition (14), also based on exogenous variables, can be interpreted as a high interest rates condition (indeed, it becomes $R^{*}>1$ in the stationary case, i.e., $e_{t}=e$, $\left.R_{t}^{*}=R^{*} \forall t\right)$. So, our result is consistent with that in Tirole (1985). The difference is that we do not require the convergence of interest rates $R_{t}^{*}$ as in Tirole (1985) or in Farhi and Tirole (2012).

Remark 2. Condition (14) is violated in a number of examples of bubbles in the literature. Indeed, in Example 1 in Kocherlakota (1992) and Example 4.2 in Santos and Woodford (1997) of fiat money, we can verify that $\lim _{t \rightarrow \infty} \frac{e_{t}}{R_{1}^{*} \cdots R_{t}^{*}}=\infty$, i.e., condition (14) is violated. Moreover, in examples of bubbles in Bosi et al. (2018b), we have $R_{t}^{*}=0$, and hence condition (14) is also violated.

Condition (14) helps us to understand better why bubbles may exist in their models. For example, Santos and Woodford (1997) claimed in page 41 that fiat money has positive value because for each household the borrowing limits effectively bind infinitely often. We go further by showing conditions (based on fundamentals) under which borrowing constraints bind.

Remark 3 (interest rates in the economy with adjusted endowments). Assume that borrowing limits are low enough so that $e_{1, t}-d_{t} b_{1}^{*}, e_{2, t}-d_{t} b_{2}^{*}$ are strictly positive. By using the same argument in Proposition 4, we can prove that there is no bubble if

$$
\lim _{t \rightarrow \infty} \frac{e_{t}}{R_{1}^{d} \cdots R_{t}^{d}}=0
$$


where $R_{t}^{d}$ is defined by

$$
1=\frac{\gamma_{1,2 t-1} u_{1}^{\prime}\left(e_{1,2 t}-d_{2 t} b_{1}^{*}\right)}{u_{1}^{\prime}\left(e_{1,2 t-1}-b_{1}^{*} d_{2 t-1}\right)} R_{2 t}^{d}, \quad 1=\frac{\gamma_{2,2 t} u_{2}^{\prime}\left(e_{2,2 t+1}-d_{2 t+1} b_{2}^{*}\right)}{u_{2}^{\prime}\left(e_{2,2 t}-b_{2}^{*} d_{2 t}\right)} R_{2 t+1}^{d}
$$

which can be interpreted as the interest rate of the economy with adjusted endowments. When there is no dividend $\left(d_{t}=0 \forall t\right)$ or agents are prevented from borrowing $\left(b_{1}^{*}=\right.$ $b_{2}^{*}=0$ ), then $R_{t}^{d}=R_{t}^{*}$.

\subsection{The set of (bubbly) equilibria}

We observe that, for $x>0$, the sequence $\left(q_{t}\right)_{t \geq 0}$, defined by $q_{0}=x$ and the system $(10)$, is unique. So, we denote this sequence by $\left(q_{t}(x)\right)_{t}$. Denote $\mathcal{S}_{0}$ the set of initial prices, i.e., the set of all values $x>0$ such that the sequence $\left(q_{t}(x)\right)$ is a sequence of prices of an equilibrium whose allocations are given by (8) and (9a-9c).

The following result shows interesting properties of the set $\mathcal{S}_{0}$.

Proposition 5. Let Assumption 1, 2 be satisfied. Assume that for $i=1,2$, the function $c u_{i}^{\prime}(c)$ is increasing in $c$, and that $e_{1, t}-d_{t} b_{1}^{*}>0, e_{2, t}-d_{t} b_{2}^{*}>0 \forall t$.

The set $\mathcal{S}_{0}$ is bounded and connected (in the sense that, if $x, y \in \mathcal{S}_{0}$ and $x<y$, then $\left.(x, y) \subset \mathcal{S}_{0}\right)$. So, if the set $\mathcal{S}_{0}$ is non-empty, either it contains a unique element or it is an interval. By consequence, we have that:

1. There is at most one bubbleless equilibrium.

2. If $\mathcal{S}_{0}$ contains at least 2 elements, there is a continuum of bubbly equilibria.

Proof. See Appendix B.

The key point of this result is that $q_{t}$ and $R_{t}$ are strictly increasing in $q_{0}$ while the fundamental value $F V_{0} \equiv \sum_{t \geq 1} Q_{t} d_{t}$ is strictly decreasing in $q_{0}$. Although Proposition 5 shows important characteristics of the set of (bubbly) equilibrium, it remains to find

conditions under which this set contains at least 2 elements. We will do so in the next subsection.

\subsection{New examples of bubbles}

In this section, we will provide new examples of bubbles. We will work under logarithmic utility functions, i.e., $u_{i}(c)=\ln (c) \forall i=1$, 2. In this case, the FOCs give

$$
\begin{cases}\frac{e_{2,1}-b_{2}^{*} d_{1}}{q_{1}+d_{1}} & =\gamma_{2,0} \frac{e_{2,0}+d_{0} b_{2,-1}}{q_{0}}-\gamma_{2,0}\left(L+b_{1}^{*}-b_{2,-1}\right)-H \\ \frac{e_{1,2 t}-d_{2 t} b_{1}^{*}}{q_{2 t}+d_{2 t}} & =\frac{\gamma_{1,2 t-1}\left(e_{1,2 t-1}-b_{1}^{*} d_{2 t-1}\right)}{q_{2 t-1}}-H\left(\gamma_{1,2 t-1}+1\right) \\ \frac{e_{2,2 t+1}-d_{2 t+1} b_{2}^{*}}{q_{2 t+1}+d_{2 t+1}} & =\frac{\gamma_{2,2 t}\left(e_{2,2 t}-b_{2}^{*} d_{2 t}\right)}{q_{2 t}}-H\left(\gamma_{2,2 t}+1\right) .\end{cases}
$$


where recall that $H \equiv L+b_{1}^{*}+b_{2}^{*}$.

It is not easy to study this system because it is non-stationary and there are infinitely many parameters. We begin our exposition by studying a specific case and then present our results in the general case.

Example 1. Assume that $\beta_{i, t}=\beta^{t}$ where $\beta \in(0,1)$ (which implies that $\gamma_{i, t}=\beta \forall t$, and $\forall i=1,2)$ and there is no dividend $\left(d_{t}=0 \forall t\right)$. Assume also that $b_{1,-1}=L+b_{2}^{*}$, $b_{2,-1}=-b_{2}^{*}$, and endowments are periodic

$$
\left(e_{1, t}\right)_{t \geq 0}=(w, e, w, e, \ldots), \quad\left(e_{2, t}\right)_{t \geq 0}=(e, w, e, w, \ldots)
$$

where $e, w>0\left(\right.$ so $\left.e_{t}=e>0, w_{t}=w>0 \forall t\right)$.

1. If $\frac{\beta e}{w} \leq 1$ (i.e., $R^{*} \geq 1$ ), there is no bubble.

2. If $\frac{\beta e}{w}>1$ (i.e., $R^{*}<1$ : low interest rate condition), then the initial price of any equilibrium with bubble must satisfy condition $q_{0} \leq \frac{1}{H} \frac{\beta e-w}{1+\beta}$. Conversely, we have:

(a) There is a unique equilibrium with initial price $q_{0}=\frac{1}{H} \frac{\beta e-w}{1+\beta}$. Moreover, this equilibrium is stationary in the sense that $q_{t}=\frac{1}{H} \frac{\beta e-w}{1+\beta}>0 \forall t$.

(b) (Continuum of equilibria with bubble) For any value $x$ in the interval $\left[0, \frac{1}{H} \frac{\beta e-w}{1+\beta}\right)$, the sequence $\left(q_{t}\right)$ determined by $q_{0}=x$ and $\frac{1}{H q_{t+1}}=\frac{\beta e}{w} \frac{1}{H q_{t}}-\frac{1+\beta}{w}$ $\forall t \geq 0,{ }^{18}$ is a system of price of an equilibrium with bubble. Moreover, (1) $q_{t}$ is decreasing in $t$ and converges to zero, (2) the interest rate $R_{t} \equiv q_{t} / q_{t-1}$ is decreasing in $t$ and converges to $R^{*}=\frac{w}{\beta e}<1$.

Proof. See Appendix B.1.

Notice that in the case of bubbles in Example 1, the seesaw property (13) holds and low interest rate condition (14) is violated (because $e_{t}=e$ and $R^{*}<1$ )

Example 1 is related to existing models of bubbles in general equilibrium, for instance, Example 4.1 in Santos and Woodford (1997), Townsend (1980), Chapter 27 in Ljungqvist and Sargent (2012) (their model corresponds to the case $e=1, w=0$ in our model), Section 2 in Bloise and Citanna (2019). Example 1 can also be viewed as a version of the main result in Tirole (1985) (Proposition 1) for an exchange general equilibrium model with infinitely lived agents and short-sale constraints. With our specification, we explicitly compute the maximum level of initial price bubble (which equals $\left.\frac{1}{H} \frac{\beta e-w}{1+\beta}\right)$ while it is implicit in more general models.

A value added of Example 1 is that it completely characterize the set of multiple equilibria. By the way, it is more general than Example 4.1 in Santos and Woodford (1997), which only examines two particular cases $q_{0}=0$ or $q_{0}=\frac{1}{H} \frac{\beta e-w}{1+\beta}$.

\footnotetext{
${ }^{18}$ By convention, if $q_{0}=0$, we determine $q_{t}=0 \forall t \geq 1$.
} 
However, Example 1 and those in the existing literature do not clearly show us how the existence of bubbles depends on the dynamics of economic fundamentals and on the asset structure (dividends and borrowing limits). In the next subsections, we will work in more general setups in order to investigate this issue.

\subsubsection{Asset without dividends}

We focus on the case of fiat money or pure bubble asset (i.e., $d_{t}=0 \forall t$ ). To simplify our exposition, we introduce some notations.

$$
\begin{aligned}
& \gamma_{2 t} \equiv \gamma_{2,2 t}=\frac{\beta_{2,2 t+1}}{\beta_{2,2 t}}, \quad \gamma_{2 t-1} \equiv \gamma_{1,2 t-1}=\frac{\beta_{1,2 t}}{\beta_{1,2 t-1}} \\
& e_{2 t} \equiv e_{2,2 t}, \quad e_{2 t-1} \equiv e_{1,2 t-1}, \quad w_{2 t} \equiv e_{1,2 t}, \quad w_{2 t-1} \equiv e_{2,2 t-1}
\end{aligned}
$$

One can verify that $\frac{\gamma_{t-1} e_{t-1}}{w_{t}}=\frac{1}{R_{t}^{*}}$. We then denote

$$
\begin{aligned}
\Gamma_{t} & \equiv \frac{\gamma_{t-1} e_{t-1}}{w_{t}} \cdots \frac{\gamma_{0} e_{0}}{w_{1}}=\frac{1}{R_{1}^{*} \cdots R_{t}^{*}} \\
D_{t} & \equiv \frac{1+\gamma_{t-1}}{w_{t}}+\frac{1}{R_{t}^{*}} \frac{1+\gamma_{t-2}}{w_{t-1}}+\cdots+\frac{1}{R_{t}^{*} \cdots R_{2}^{*}} \frac{1+\gamma_{0} \frac{L+b_{1}^{*}-b_{2,-1}}{L+b_{1}^{*}+b_{2}^{*}}}{w_{1}}
\end{aligned}
$$

Note that $\Gamma_{t}$ is exogenous and $\Gamma_{t} \geq Q_{t}=\frac{1}{R_{1} \cdots R_{t}} \forall t \geq 2$ because $R_{t} \geq R_{t}^{*} \forall t \geq 2$.

The following result provides necessary and sufficient conditions under which bubbles exist in equilibrium.

Proposition 6 (continuum of equilibria with bubble). Assume that $d_{t}=0 \forall t$ and $u_{i}(c)=\ln (c) \forall i=1,2$.

1. (Necessary condition) If the sequence $\left(q_{t}\right)_{t \geq 0}$, asset holdings given by (8) and agents' consumptions given by (9a-9c) constitute an equilibrium with bubble, then we have

$$
\frac{1}{H q_{t}}=\frac{1}{H q_{0}} \Gamma_{t}-D_{t} \forall t
$$

By consequence, we have $q_{0} \leq \frac{\Gamma_{t}}{H D_{t}} \forall t$ and therefore

$$
\sup _{t}\left(\frac{H D_{t}}{\Gamma_{t}}\right)<\infty \text { and } \sum_{t=1}^{\infty} \frac{R_{1}^{*} \cdots R_{t}^{*}}{e_{t}}<\infty
$$

2. (Sufficient condition to have a continuum of equilibria with bubbles) Assume, in addition, that $\gamma_{1,2 t+1} \geq \gamma_{2,2 t+1}, \gamma_{2,2 t} \geq \gamma_{1,2 t}, e_{t}>w_{t}$ (i.e., $e_{2,2 t}>e_{1,2 t}$, $\left.e_{1,2 t+1}>e_{2,2 t+1}\right) \forall t$. 
If

$$
\sup _{t} \frac{H\left(D_{t}+\frac{2}{e_{t}-w_{t}}\right)}{\Gamma_{t}}<\infty
$$

then any sequence $\left(q_{t}\right)_{t \geq 0}$ determined by

$$
\begin{aligned}
& \qquad q_{0} \in(0, \bar{q}), \quad \frac{1}{H q_{t}}=\frac{1}{H q_{0}} \Gamma_{t}-D_{t} \forall t \geq 1 \\
& \text { where } \bar{q} \equiv \min \left\{\inf _{t \geq 1}\left(\frac{\Gamma_{t}}{H\left(D_{t}+\frac{2}{e_{t}-w_{t}}\right)}\right), \frac{e_{2,0}-e_{1,0}}{L+2 b_{1}^{*}+b_{1,-1}-b_{2,-1}}\right\}>0
\end{aligned}
$$

is a system of prices of an equilibrium at which asset holdings are given by (8) and agents' consumptions are given by (9a-9c). Moreover, all such equilibria are bubbly.

Proof. See Appendix B.1.

According to condition (22), interest rates of the economy without asset must be low. Notice that condition (22) also implies that $\lim _{t \rightarrow \infty} \frac{e_{t}}{R_{1}^{*} \cdots R_{t}^{*}}=\infty$, i.e., the present value of endowment of the autarkic economy is infinite. It means that the no-bubble condition (14) in Proposition 4 is violated.

Condition (23) is a key in Proposition 6. We can compute that

$$
\begin{aligned}
\frac{H\left(D_{t}+\frac{2}{e_{t}-w_{t}}\right)}{\Gamma_{t}}= & \frac{H w_{1} \cdots w_{t-1}}{e_{0} \cdots e_{t-1}} \frac{1+\gamma_{t-1}}{\gamma_{0} \cdots \gamma_{t-1}}+\frac{H w_{1} \cdots w_{t-2}}{e_{0} \cdots e_{t-2}} \frac{1+\gamma_{t-2}}{\gamma_{0} \cdots \gamma_{t-2}}+ \\
& +\cdots+\frac{H}{e_{0}}\left(\frac{1}{\gamma_{0}}+\frac{L+b_{1}^{*}-b_{2,-1}}{L+b_{1}^{*}+b_{2}^{*}}\right)+\frac{2 H w_{1} \cdots w_{t}}{e_{0} \cdots e_{t-1}\left(e_{t}-w_{t}\right)} \frac{1}{\gamma_{0} \cdots \gamma_{t-1}} .
\end{aligned}
$$

So, (23) can be satisfied for a large class of parameters (for example, $\gamma_{t}=\gamma \in(0,1)$ and $w_{t}=e_{t} x$ where $\left.x \in(0, \gamma)\right)$.

Thanks to condition (23), the value $\bar{q}$ defined by (24b) is strictly positive. Proposition 6 suggests that when the economy without assets has low interest rates (in the sense that condition (23) holds), an equilibrium with binding borrowing constraints has bubbles if the initial price $q_{0}$ is low enough in the sense that $q_{0} \leq \bar{q}$. It is useful to understand how the upper bound $\bar{q}$ depends on fundamentals. According to (25), we observe that $\bar{q}$ is decreasing in the asset supply $L$, borrowing limits $b_{1}^{*}, b_{2}^{*}$, the endowment ratio $\frac{w_{t}}{e_{t}}$, the initial asset holding $b_{1,-1}$ of agent 1 , and $\bar{q}$ is increasing in the rate of time preference $\gamma_{t}$, the initial asset holding $b_{2,-1}$ of agent 2 .

To sum up, the existence of bubble requires low interest rates and seesaw property of the economy without asset. Moreover, when these necessary conditions hold, bubbles are more likely to exist if

1. Asset supply $L$ is low. (Asset shortage.) 
2. Borrowing limits $b_{1}^{*}$ and $b_{2}^{*}$ are low. (Financial frictions matter.)

3. The initial asset $b_{2,-1}$ is high and/or the initial asset $b_{1,-1}$ is low. (Heterogeneity matters.)

4. The endowment ratios $\frac{e_{2,2 t}}{e_{1,2 t}}$ and $\frac{e_{1,2 t+1}}{e_{2,2 t+1}}$ are high. (Heterogeneity and seesaw property.)

5. The rates of time preference $\frac{\beta_{2,2 t+1}}{\beta_{2,2 t}}$ and $\frac{\beta_{1,2 t}}{\beta_{1,2 t-1}}$ are high (heterogeneity and seesaw property).

When $\beta_{i, t}=\beta^{t} \forall i, t$, the existence of bubbles requires that $\beta$ must be high enough (this is consistent with the finding in Proposition 3).

Equilibrium indeterminacy and bubbles. Proposition 6 and Example 1 show that, not only asset price bubbles but also real indeterminacy exist. Indeed, in equilibrium, the agent 2 buys asset at date $2 t\left(b_{2,2 t}=L+b_{1}^{*}\right)$ and the agent 1 buys asset at date $2 t+1\left(b_{1,2 t+1}=L+b_{2}^{*}\right)$. Consumptions are given by

$$
\begin{aligned}
c_{1,0} & =e_{1,0}+q_{0}\left(b_{1,-1}+b_{a}^{*}\right), & c_{2,0}=e_{2,0}+q_{0}\left(b_{2,-1}-L-b_{a}^{*}\right) \\
c_{1,2 t} & =e_{1,2 t}+q_{2 t} H, & c_{2,2 t}=e_{2,2 t}-q_{2 t} H \\
c_{1,2 t+1} & =e_{1,2 t+1}-q_{2 t+1} H, & c_{2,2 t+1}=e_{2,2 t+1}+q_{2 t+1} H .
\end{aligned}
$$

where the sequence of prices $\left(q_{t}\right)$ is determined by $(24 \mathrm{a})$ and $(24 \mathrm{~b})$. Since there is a continuum of equilibrium price systems, there is real indeterminacy. This point is interesting because our model contains only one consumption good and a single asset. Our framework indicates that financial frictions and heterogeneity may generate real indeterminacy.

Recall that $q_{t}$ is increasing in $q_{t-1}$ and hence in the initial price $q_{0}$. So, for any $t \geq$ 1 , we observe that (1) the consumptions $c_{1,2 t}$ is increasing in $q_{0}$ but $c_{1,2 t-1}$ decreasing in $q_{0}$ and $(2) c_{2,2 t}$ is decreasing in $q_{0}$ but $c_{2,2 t-1}$ is increasing in $q_{0}$.

We also observe that the presence of multiple asset prices may affect the consumption inequality. Indeed, we have $\frac{c_{1,2 t}}{c_{2,2 t}}$ is increasing in $q_{2 t}$ and so is in $q_{0}$. By contrast, the ratio $\frac{c_{1,2 t+1}}{c_{2,2 t+1}}$ is decreasing in $q_{2 t+1}$ and so is in $q_{0}$.

The number of agents matters. Assume that there are $n_{1}$ agents of type $A$ and $n_{2}$ agents of type 2 . For the sake of simplicity, we assume that $n_{1}=n_{2}=n$ ( there are $m=2 n$ agents in the economy). In this case, the asset holding of agents is

$$
\begin{aligned}
b_{1,2 t} & =-b_{1}^{*}, \quad b_{2,2 t}=\frac{L+n b_{1}^{*}}{n}=\frac{L}{n}+b_{1}^{*}, \quad b_{1,2 t+1}=\frac{L}{n}+b_{2}^{*}, \quad b_{2,2 t+1}=-b_{2}^{*} \\
L_{n} & \equiv \frac{L}{n}, \quad H_{n} \equiv L_{n}+b_{1}^{*}+b_{2}^{*}
\end{aligned}
$$


With these asset holdings, we have that

$$
\begin{aligned}
c_{1,0} & =e_{1,0}+\left(q_{0}+d_{0}\right) b_{1,-1}+q_{0} b_{1}^{*}, \quad c_{2,0}=e_{2,0}+\left(q_{0}+d_{0}\right) b_{2,-1}-q_{0}\left(L_{n}+b_{1}^{*}\right) \\
c_{1,2 t-1} & =e_{1,2 t-1}-b_{1}^{*} d_{2 t-1}-q_{2 t-1} H_{n}, \quad c_{2,2 t-1}=e_{2,2 t-1}+d_{2 t-1}\left(L_{n}+b_{1}^{*}\right)+q_{2 t-1} H_{n} \\
c_{1,2 t} & =e_{1,2 t}+d_{2 t}\left(L_{n}+b_{2}^{*}\right)+q_{2 t} H_{n}, \quad c_{2,2 t}=e_{2,2 t}-d_{2 t} b_{2}^{*}-q_{2 t} H_{n}
\end{aligned}
$$

By applying our above results, bubbles are more likely to exist when $L_{n}=L / n$ is low (i.e., the number of agents is high).

\subsubsection{Assets with positive dividends}

In this subsection, we study the existence and dynamics of bubbles of assets having positive dividends (i.e., $d_{t}>0 \forall t \geq 0$ ). Note that there are very few papers providing examples of this kind of bubble. ${ }^{19}$

Consider a general model, according to the asset pricing equation $q_{t}=\frac{q_{t+1}+d_{t+1}}{R_{t+1}}$, we have $q_{t} Q_{t}=q_{t+1} Q_{t+1}\left(1+\frac{d_{t+1}}{q_{t+1}}\right)$. By iterating, we get that $q_{0}=q_{T} Q_{T} \prod_{t=1}^{T}\left(1+\frac{d_{t}}{q_{t}}\right)$. Bubbles exist (i.e., $\lim _{t \rightarrow} Q_{t} q_{t}>0$ ) if and only if $\lim _{t \rightarrow \infty} \prod_{t=1}^{T}\left(1+\frac{d_{t}}{q_{t}}\right)<\infty$, or equivalently

$$
\sum_{t} \frac{d_{t}}{q_{t}}<\infty
$$

This means that there is a bubble if the price $q_{t}$ goes faster than the dividend $d_{t}{ }^{20}$ By consequence, we obtain the following result.

Corollary 3. Consider an equilibrium whose allocations given by (8) and (9a-9c). The existence of bubble implies that

$$
\sum_{t} \frac{d_{2 t}}{e_{2,2 t}-b_{2}^{*} d_{2 t}}<\infty \text { and } \sum_{t} \frac{d_{2 t-1}}{e_{1,2 t-1}-b_{1}^{*} d_{2 t-1}}<\infty .
$$

This means that the existence of bubbles in equilibrium requires a low level of dividends with respect to the agents' endowment in the future. ${ }^{21}$ The intuition behind is that, the existence of bubble requires that the asset price goes faster than the dividend. Since trading takes place at every date, the income of asset buyers and hence their endowments must go faster than the dividends.

\footnotetext{
${ }^{19}$ See Le Van and Pham (2016) Bosi et al. (2017a, 2018b), Bloise and Citanna (2019).

${ }^{20}$ This condition was also proved in Montrucchio (2004), Bosi et al. (2018b).

${ }^{21}$ This condition is consistent with but stronger than no-bubble condition $\liminf _{t} \frac{d_{t}}{\sum_{i} e_{i, t}}>0$ in Corollary 2.
} 
Before providing conditions under which there is a continuum of bubbly equilibria, we introduce some notations.

$$
\left\{\begin{array} { l } 
{ a _ { 1 } \equiv \frac { \gamma _ { 2 , 0 } ( e _ { 2 , 0 } + d _ { 0 } b _ { 2 , - 1 } ) } { e _ { 2 , 1 } - b _ { 2 } ^ { * } d _ { 1 } } } \\
{ a _ { 2 t } \equiv \frac { \gamma _ { 1 , 2 t - 1 } ( e _ { 1 , 2 t - 1 } - b _ { 1 } ^ { * } d _ { 2 t - 1 } ) } { e _ { 1 , 2 t } - b _ { 1 } ^ { * } d _ { 2 t } } } \\
{ a _ { 2 t + 1 } \equiv \frac { \gamma _ { 2 , 2 t } ( e _ { 2 , 2 t } - b _ { 2 } ^ { * } d _ { 2 t } ) } { e _ { 2 , 2 t + 1 } - b _ { 2 } ^ { * } d _ { 2 t + 1 } } }
\end{array} \quad \left\{\begin{array} { l } 
{ H _ { 1 } \equiv \frac { \gamma _ { 2 , 0 } ( L + b _ { 1 } ^ { * } - b _ { 2 , - 1 } ) + H } { e _ { 2 , 1 } - b _ { 2 } ^ { * } d _ { 1 } } } \\
{ H _ { 2 t } \equiv \frac { H ( 1 + \gamma _ { 1 , 2 t - 1 } ) } { e _ { 1 , 2 t } - b _ { 1 } ^ { * } d _ { 2 t } } } \\
{ H _ { 2 t + 1 } \equiv \frac { H ( 1 + \gamma _ { 2 , 2 t } ) } { e _ { 2 , 2 t + 1 } - b _ { 2 } ^ { * } d _ { 2 t + 1 } } }
\end{array} \quad \left\{\begin{array}{l}
\bar{q}_{0} \equiv \frac{e_{2,0}-e_{1,0}-d_{0}\left(b_{1,-1}-b_{2,-1}\right)}{L+2 b_{1}^{*}+b_{1,-1}-b_{2,-1}} \\
\bar{q}_{2 t-1} \equiv \frac{e_{1,2 t-1}-e_{2,2 t-1}-\left(L+2 b_{1}^{*}\right) d_{2 t-1}}{2 H} \\
\bar{q}_{2 t} \equiv \frac{e_{2,2 t}-e_{1,2 t}-\left(L+2 b_{2}^{*}\right) d_{2 t}}{2 H}
\end{array}\right.\right.\right.
$$

Recall that $\left(R_{t}^{d}\right)$-the interest rates of the economy with adjusted endowments- defined by (16) satisfies $1=a_{t} R_{t}^{d}$.

The FOCs (17) can be rewritten as

$$
\frac{1}{q_{t}+d_{t}}=\frac{a_{t}}{q_{t-1}}-H_{t} \forall t \geq 1 \text {, or equivalently } q_{t}=\frac{q_{t-1}}{a_{t}-H_{t} q_{t-1}}-d_{t} \forall t \geq 1
$$

If $\left(q_{t}\right)$ is a sequence of price, we must have

$$
\frac{a_{t} d_{t}}{1+d_{t} H_{t}}<q_{t-1}<\frac{a_{t}}{H_{t}} \forall t \geq 1
$$

So, the equilibrium price at each date must be bounded by exogenous values.

We now state the main result in this section, which shows that bubbles may exist under strong heterogeneity and low dividends.

Proposition 7 (multiple equilibria with bubbles). Let $u_{i}(c)=\ln (c) \forall i=1,2$. Assume that $H_{t}>0, a_{t+1} / H_{t+1}<\bar{q}_{t} \forall t$ and there are sequences $\left(\alpha_{t}\right)_{t \geq 1},\left(\sigma_{t}\right)_{t \geq 1}$ such

$$
0<\alpha_{t}<1<\sigma_{t}
$$

$$
\begin{aligned}
\text { Strong heterogeneity and seesaw property: } & a_{t+1}>\frac{H_{t+1}}{H_{t}} \frac{\alpha_{t}}{\alpha_{t+1}\left(1-\alpha_{t}\right)} \\
\text { Low dividend condition: } & \left\{\begin{array}{c}
\frac{d_{t}}{d_{t+1}}>\frac{\sigma_{t+1}}{\sigma_{t}-1} a_{t+1} \\
1-\left(\sigma_{t}-1\right) d_{t} H_{t}>0 \\
\text { and } \frac{\sigma_{1} a_{1} d_{1}}{1+d_{1} H_{1}}<\frac{\alpha_{1} a_{1}}{H_{1}}
\end{array}\right.
\end{aligned}
$$

Then, there is a continuum of bubbly equilibria. More precisely, any sequence $\left(q_{t}\right)_{t \geq 0}$ determined by

$$
\left\{\begin{array}{l}
q_{0} \in\left(\frac{\sigma_{1} a_{1} d_{1}}{1+d_{1} H_{1}}, \frac{\alpha_{1} a_{1}}{H_{1}}\right) \\
\text { and the system (17) }
\end{array}\right.
$$

is a system of prices of an equilibrium in which asset holdings are given by (8) and agents' consumptions are given by (9a-9c). Moreover for such equilibrium, we have

$$
\frac{\sigma_{t} a_{t} d_{t}}{1+d_{t} H_{t}}<q_{t-1}<\frac{\alpha_{t} a_{t}}{H_{t}} \forall t \geq 1
$$


Proof. See Appendix B.2.

Remark 4 (heterogeneity, seesaw property and low interest rate condition). We interpret condition (32b) as a strong heterogeneity and a seesaw property because we observe that

$$
\begin{aligned}
& \frac{a_{2 t+1} H_{2 t}}{H_{2 t+1}}=\frac{\gamma_{2,2 t}\left(1+\gamma_{1,2 t-1}\right)}{1+\gamma_{2,2 t}} \frac{e_{2,2 t}-b_{2}^{*} d_{2 t}}{e_{1,2 t}-b_{1}^{*} d_{2 t}} \\
& \frac{a_{2 t} H_{2 t-1}}{H_{2 t}}=\frac{\gamma_{1,2 t-1}\left(1+\gamma_{2,2 t-2}\right)}{1+\gamma_{1,2 t-1}} \frac{e_{1,2 t-1}-b_{1}^{*} d_{2 t-1}}{e_{2,2 t-1}-b_{2}^{*} d_{2 t-1}} .
\end{aligned}
$$

Since the interest rates of the economy with adjusted endowments are $R_{t}^{d}=1 / a_{t} \forall t$, condition (32b) can also be interpreted as a "low interest rate condition".

Proposition 7 is a generalization of Proposition 6. To the best of our knowledge, Proposition 7 is the first result showing the existence of multiple equilibria with bubbles of assets with positive dividends in deterministic general equilibrium models. ${ }^{22}$ Note that dividends and endowments are not necessarily stationary.

It is important to mention that there exist exogenous parameters satisfying all conditions in Proposition 7. Indeed, we can choose parameters as follows.

1. Choose $\alpha_{t}=\alpha, \sigma_{t}=\sigma \forall t$.

2. Choose $\gamma_{i, t}=\beta \in(0,1) \forall i, \forall t$. In this case, we have

$$
\frac{a_{1}}{H_{1}}=\frac{\beta\left(e_{2,0}+d_{0} b_{2,-1}\right)}{\beta\left(L+b_{1}^{*}-b_{2,-1}\right)+H}, \frac{a_{2 t}}{H_{2 t}}=\frac{\beta\left(e_{1,2 t-1}-b_{1}^{*} d_{2 t-1}\right)}{(1+\beta) H}, \frac{a_{2 t+1}}{H_{2 t+1}}=\frac{\beta\left(e_{2,2 t}-b_{2}^{*} d_{2 t}\right)}{(1+\beta) H}
$$

So, condition $\frac{a_{t+1}}{H_{t+1}}<\bar{q}_{t}$ is equivalent to

$$
\begin{aligned}
\frac{\beta\left(e_{2,0}+d_{0} b_{2,-1}\right)}{\beta\left(L+b_{1}^{*}-b_{2,-1}\right)+H} & <\frac{e_{2,0}-e_{1,0}-d_{0}\left(b_{1,-1}-b_{2,-1}\right)}{L+2 b_{1}^{*}+b_{1,-1}-b_{2,-1}} \\
\frac{\beta\left(e_{1,2 t-1}-b_{1}^{*} d_{2 t-1}\right)}{(1+\beta) H} & <\frac{e_{1,2 t-1}-e_{2,2 t-1}-\left(L+2 b_{1}^{*}\right) d_{2 t-1}}{2 H} \\
\frac{\beta\left(e_{2,2 t}-b_{2}^{*} d_{2 t}\right)}{(1+\beta) H} & <\frac{e_{2,2 t}-e_{1,2 t}-\left(L+2 b_{2}^{*}\right) d_{2 t}}{2 H} .
\end{aligned}
$$

3. Choose $e_{2,2 t+1}, e_{1,2 t}$ such that $H_{t}=h>0 \forall t$. Hence, $\frac{H_{t+1}}{H_{t}}=1$.

\footnotetext{
${ }^{22}$ Le Van and Pham (2016) (Section 6.1) and Bosi et al. (2017a) provide examples of bubbles of the Lucas' tree, where the asset price may be multiple (due to the portfolio effect) but the consumption is not affected by the existence of bubbles. Our added-value is that the equilibrium indeterminacy in our model is real (in the sense that different equilibria have different consumption allocations) and the asset price affects agents' consumptions.
} 
4. Given that $\left(d_{t}\right)$ is low, we can choose $e_{2,2 t}, e_{1,2 t+1}$ sufficiently high so that (1$\alpha) a_{t+1}>1$ and (34a-34c) hold. (This is a low interest rates condition.)

5. Choose $\left(d_{t}\right)$ and $\frac{d_{t+1}}{d_{t}}$ low enough such that $(32 \mathrm{~b}),(32 \mathrm{c})$ are satisfied and $\frac{\sigma a_{1} d_{1}}{1+d_{1} H_{1}}<$ $\frac{\alpha a_{1}}{H_{1}}$. (This is a low dividend condition.)

Although Proposition 7 provides a general sufficient condition under which there is a continuum of equilibria with bubbles, it would be useful to give examples with explicit mechanisms. We firstly focus on parameters satisfying the following assumption.

Assumption 4. Assume that $\gamma_{i, t}=\beta \in(0,1)$ (i.e., $\beta_{i, t}=\beta^{t}$ ) and endowments are

$$
e_{1,2 t-1}=b_{1}^{*} d_{2 t-1}+e, \quad e_{1,2 t}=b_{1}^{*} d_{2 t}+w, \quad e_{2,2 t-1}=b_{2}^{*} d_{2 t-1}+w, \quad e_{2,2 t}=b_{2}^{*} d_{2 t}+e
$$

where $e, w>0$.

Under this specification, $\left(a_{t}\right),\left(H_{t}\right)$ defined by (29) become $a_{t}=a=\frac{\beta e}{w}, H_{t}=h \equiv$ $\frac{H(\beta+1)}{w} \forall t$, and the system of price $\left(q_{t}\right)$ satisfies

$$
\frac{1}{q_{t}+d_{t}}=\frac{a}{q_{t-1}}-h \forall t \geq 1 \text {, or equivalently } q_{t}=\frac{q_{t-1}}{a-h q_{t-1}}-d_{t} \forall t \geq 1
$$

In this case, we have the following result which helps us to identify all possible outcomes of equilibrium.

Proposition 8. Let $u_{i}(c)=\ln (c) \forall i=1,2$ and Assumption 4 be satisfied. Assume that $\left(q_{t}\right)$ is the price of an equilibrium in which asset holdings are given by (8) and agents' consumptions are given by (9a-9c).

1. If $\frac{\beta e}{w}<1$ (i.e., $R^{*}>1$ : the interest rate of the benchmark economy is high), then there is no bubble.

2. If $\frac{\beta e}{w}>1$ (i.e., $R^{*}<1$ : the interest rate of the benchmark economy is low), then there are only three cases:

(a) There is no bubble.

(b) The equilibrium is bubbly and $q_{t}$ converges to zero.

(c) The equilibrium is bubbly, $q_{t}>\frac{\frac{\beta e}{w}-1}{\frac{H(\beta+1)}{w}} \forall t$, and $q_{t}$ converges to $\frac{\frac{\beta e}{w}-1}{\frac{H(\beta+1)}{w}}$.

Moreover, when $\frac{\beta e}{w}>1$, there is almost one equilibrium satisfying conditions (8), $(9 a-9 c)$ and $q_{t}>\frac{\frac{\beta e}{w}-1}{\frac{H(\beta+1)}{w}} \forall t$.

Proof. See Appendix B.2. 
According to Proposition 8, in equilibrium with bubbles, the asset price $q_{t}$ converges either to zero or to $\frac{\frac{\beta e}{w}-1}{\frac{H(\beta+1)}{w}} \cdot{ }^{23}$

We now complement the general results of Proposition 8 by providing examples of bubbles for each case. We start by the case where $q_{t}$ converges to zero or to a positive value.

Example 2 (continuum of equilibria with bubble and $q_{t} \rightarrow 0$ ). Let $u_{i}(c)=\ln (c)$ $\forall i=1,2$ and Assumption 4 be satisfied. Assume that there exists $\sigma$ such that $1<\sigma$ and

$$
\begin{aligned}
& \text { Low interest rate condition: } \frac{\beta e}{w}>1 \\
& \text { Low dividend condition: }\left\{\begin{array}{l}
\frac{\sigma-1}{\sigma} \frac{d_{t}}{d_{t+1}}>\frac{\beta e}{w} \\
d_{t}<\frac{w}{(\sigma-1)(\beta+1) H} \\
d_{t}<\frac{\frac{1-\beta}{1+\beta} e-w}{H} \\
\frac{\sigma a d_{1}}{1+d_{1} \frac{H(\beta+1)}{w}}<\frac{\beta e-w}{H(\beta+1)}
\end{array}\right. \\
& \text { and } \frac{\beta\left(e_{2,0}+d_{0} b_{2,-1}\right)}{\beta\left(L+b_{1}^{*}-b_{2,-1}\right)+H}<\frac{e_{2,0}-e_{1,0}-d_{0}\left(b_{1,-1}-b_{2,-1}\right)}{L+2 b_{1}^{*}+b_{1,-1}-b_{2,-1}}
\end{aligned}
$$

Then, any sequence $\left(q_{t}\right)_{t \geq 0}$ determined by the system (30) and

$$
q_{0} \in\left(\frac{\sigma a d_{1}}{1+d_{1} h}, \frac{a-1}{h}\right]
$$

is a system of prices of an equilibrium at which asset holdings are given by (8) and agents' consumptions are given by (9a-9c). Moreover, Proposition 5 implies that there is a continuum of bubbly equilibria. For any equilibrium with $q_{0} \leq \frac{a-1}{h}$ (including bubbly equilibrium), the asset price $q_{t}$ decreasingly converges to zero when $t$ tends to infinity.

Example 3 (an equilibrium with bubble and $q_{t} \rightarrow q>0$ ). Let $u_{i}(c)=\ln (c) \forall i=1,2$ and Assumption 4 be satisfied. Assume also that $a>1$. Let $x>0$ such that $\frac{x+1}{x}>a>1$ and define the sequence $\left(d_{t}\right)$ by

$$
\begin{array}{r}
\frac{1}{d_{t}}=\left(\frac{x+1}{x a}\right)^{t}\left(\frac{1}{d_{0}}-\frac{h x(x+1)}{1-(a-1) x}\right)+\frac{h x(x+1)}{1-(a-1) x} \\
0<d_{0}<\frac{1-(a-1) x}{h x(x+1)}, \quad d_{0}<\frac{\frac{1-\beta}{1+\beta} e-w}{H}
\end{array}
$$

\footnotetext{
${ }^{23}$ This result is related to Propositions 2 and 3 in Bosi et al. (2018a). The difference is that Bosi et al. (2018a) consider an OLG model with descending altruism while we study a general equilibrium model with infinitely lived agents.
} 
Observe that $0<h x d_{t}<1 \forall t$ and $x d_{t}+d_{t}=\frac{a x d_{t-1}}{1-h x d_{t-1}}$. Moreover, $\sum_{t} d_{t}<\infty$.

Define the sequence $\left(q_{t}\right)$ by $q_{t}=\frac{a-1}{h}+x d_{t} \forall t \geq 0$. Then $\left(q_{t}\right)$ is a system of prices of an equilibrium at which asset holdings are given by (8) and agents' consumptions are given by (9a-9c). Moreover, $q_{t}$ decreasingly converges to $(a-1) / h$.

In this equilibrium, we have $\sum_{t}\left(d_{t} / q_{t}\right)=\sum_{t}\left(\frac{d_{t}}{\frac{a-1}{h}+x d_{t}}\right)<\sum_{t} d_{t} \frac{h}{a-1}<\infty$. So, this equilibrium experiences a bubble.

Proof. See Appendix B.2.

Let us explain the basic intuition of our Examples 2, 3. By definition (29) of $a_{t}$, condition $a=\beta e / w>1$ (i.e., the interest rate $R^{*}$ of the benchmark economy is low) is equivalent to

$$
\frac{\beta\left(e_{1,2 t-1}-b_{1}^{*} d_{2 t-1}\right)}{e_{1,2 t}-b_{1}^{*} d_{2 t}}>1 \text { and } \frac{\beta\left(e_{2,2 t}-b_{2}^{*} d_{2 t}\right)}{e_{2,2 t+1}-b_{2}^{*} d_{2 t+1}}>1 \forall t
$$

We can interpret that agent 1 is richer than agent 2 at date $2 t-1$ but agent 2 is richer than agent 1 at date $2 t$; note that this is consistent with the seesaw property (13). Hence, agent 1 (resp., agent 2) may accept to buy the financial asset at date $2 t-1$ (resp., date $2 t$ ) even the asset price is higher than the fundamental value (i.e., there is a bubble). In both Examples 2, 3, we design that the sequence of dividends is low enough in order to ensure that, for any $i, t$, the asset value $q_{t} b_{i, t}$ is lower than the resource of agent $i$ at date $t$ so that agent $i$ can buy the financial asset.

In Example 2, there is a continuum of equilibrium prices but any sequence of price converges to zero. In Example 3, we have $q_{0}=(a-1) / h$ and the sequence of prices converges to $(a-1) / h>0$ (notice that, according to Proposition 8 , this is the unique bubbly equilibrium such that $q_{t}$ converges to a strictly positive value.)

In Examples 2 and 3, the aggregate endowment is uniformly bounded and the sequence of dividends converges to zero. The following result shows that, in an economy with unbounded and asymmetric growth, bubbles may exist and the asset price may go to infinity.

Example 4 (asymmetric growth and multiple equilibria with $q_{t} \rightarrow \infty$ ). Let $u_{i}(c)=$ $\ln (c) \forall i=1,2, \gamma_{i, t}=\beta \in(0,1)$ (i.e., $\beta_{i, t}=\beta^{t}$ ). Assume that $d_{t}=d>0 \forall t$ and endowments are

$$
\begin{aligned}
& e_{1,2 t-1}=b_{1}^{*} d_{2 t-1}+e_{2 t-1}, \quad e_{1,2 t}=b_{1}^{*} d_{2 t}+w_{2 t} \\
& e_{2,2 t-1}=b_{2}^{*} d_{2 t-1}+w_{2 t-1}, \quad e_{2,2 t}=b_{2}^{*} d_{2 t}+e_{2 t}
\end{aligned}
$$


Let $\alpha$ and $\sigma$ be such that $0<\alpha<1<\sigma$. Assume that, for any $t$,

$$
\begin{aligned}
\frac{e_{2,0}-e_{1,0}-d_{0}\left(b_{1,-1}-b_{2,-1}\right)}{L+2 b_{1}^{*}+b_{1,-1}-b_{2,-1}} & >\frac{\beta\left(e_{2,0}+d_{0} b_{2,-1}\right)}{\beta\left(L+b_{1}^{*}-b_{2,-1}\right)+H} \\
\frac{1-\beta}{1+\beta} e_{t}-w_{t} & >H d \\
w_{t+1} & >\frac{\sigma}{\sigma-1} \beta e_{t}, \quad e_{t}>\frac{1}{\beta(1-\alpha)} w_{t}, \\
w_{t} & >(\sigma-1) H(\beta+1) d .
\end{aligned}
$$

Notice that the two first conditions ensure that $\frac{a_{t+1}}{H_{t+1}}<\bar{q}_{t} \forall t$.

According to Proposition 7 , any sequence $\left(q_{t}\right)_{t \geq 0}$ determined by the system (30) and $q_{0} \in\left(\frac{\sigma a_{1} d_{1}}{1+d_{1} H_{1}}, \frac{\alpha a_{1}}{H_{1}}\right)$, is a system of prices of an equilibrium in which asset holdings are given by (8) and agents' consumptions are given by (9a-9c). By consequence, Proposition 5 implies that there is a continuum of bubbly equilibria.

In this example, endowments of both agents go to infinity. However, there is an asymmetric growth: $\frac{e_{t}}{w_{t}}>\frac{1}{\beta(1-\alpha)}>1$, or equivalently $\frac{e_{1,2 t-1}-b_{1}^{*} d_{2 t-1}}{e_{2,2 t-1}-b_{2}^{*} d_{2 t-1}}>\frac{1}{\beta(1-\alpha)}$ and $\frac{e_{2,2 t}-b_{2}^{*} d_{2 t}}{e_{1,2 t}-b_{1}^{*} d_{2 t}}>\frac{1}{\beta(1-\alpha)} \forall t$. The basic intuition of bubble in this example is consistent with that in Examples 2 and 3. Indeed, agent 1 is richer than agent 2 at date $2 t-1$ but agent 2 is richer than agent 1 at date $2 t$. Hence, agent 1 (resp., agent 2) accepts to buy the financial asset at date $2 t-1$ (resp., date $2 t$ ) even the asset price contains a bubble. Moreover, the price $q_{t}$ goes to infinity when $t$ tends to infinity because endowments of both agents grow without bound.

Example 5 (an equilibrium with bubbles and $q_{t}$ may fluctuate over time). Consider a particular case where $\beta_{i, t}=\beta^{t} \forall i, \forall t$ where $\beta \in(0,1), b_{1}^{*}=b_{2}^{*}=0$ (no short-sales) and $e_{2,2 t+1}=e_{1,2 t}=0$. In this case, $\gamma_{2,2 t}=\gamma_{1,2 t-1}=\beta, H=L$ and there is a unique equilibrium satisfying condition (8)

$$
q_{2 t}=\frac{\beta}{(1+\beta) L} e_{2,2 t} \text { and } q_{2 t-1}=\frac{\beta}{(1+\beta) L} e_{1,2 t-1} .
$$

In other words, the set $\mathcal{S}_{0}$ contains a unique element. This equilibrium experiences a bubble iff $\sum_{t} d_{t} / q_{t}<\infty$ which now becomes $\sum_{t} \frac{d_{2 t}}{e_{2,2 t}}+\sum_{t} \frac{d_{2 t-1}}{e_{1,2 t-1}}<\infty$. So, we recover $(28 \mathrm{a}){ }^{24}$

It is interesting to notice that, in our example there is no causal connection between the monotonicity of the sequence of price $\left(q_{t}\right)$ and the existence of bubble. The fact that the price $q_{t}$ increases or decreases in time does not depend on the existence of bubble but depends on the dynamics of endowments.

\footnotetext{
${ }^{24}$ This corresponds to the key condition for bubbles in Section 5.1.1 in Bosi et al. (2018b)
} 
We now look at the consumption in our example.

$$
\begin{aligned}
c_{1,0} & =e_{1,0}+\left(q_{0}+d_{0}\right) b_{1,-1}, \quad c_{2,0}=e_{2,0}+\left(q_{0}+d_{0}\right) b_{2,-1}-q_{0} L \\
c_{1,2 t-1} & =e_{1,2 t-1}-q_{2 t-1} L, \quad c_{2,2 t-1}=e_{2,2 t-1}+d_{2 t-1} L+q_{2 t-1} L \\
c_{1,2 t} & =e_{1,2 t}+d_{2 t} L+q_{2 t} L, \quad c_{2,2 t}=e_{2,2 t}-q_{2 t} L
\end{aligned}
$$

Since $L q_{2 t}=\frac{\beta}{1+\beta} e_{2,2 t}$ and $L q_{2 t-1}=\frac{\beta}{1+\beta} e_{1,2 t-1}$, we see that $c_{1,2 t-1}$ and $c_{2,2 t}$ do not depend on $\left(d_{t}\right)_{t}$ but $c_{1,2 t}$ (resp., $c_{2,2 t-1}$ ) is strictly increasing in $d_{2 t}$ (resp., $d_{2 t-1}$ ). So, when dividends decrease, bubbles will be more likely to exist but the individual welfares will be lower.

\subsection{Welfare analysis}

It would be important to compare the individual welfares generated by different equilibria. The following result allows us to do so.

Proposition 9. Consider equilibria satisfying conditions (8) and (9a-9c). Since equilibrium outcomes can be uniquely computed from the initial price $q_{0}$, the individual welfare of agent $i$ is a function of $q_{0}$, and so denoted by $W_{i}\left(q_{0}\right)$.

Assume that the utility function $u_{i}$ is differentiable and strictly concave $\left(u_{i}^{\prime \prime}<0\right)$ for any $i=1,2$. Then, we have that:

1. For any $i=1,2$, the individual welfare $W_{i}\left(q_{0}\right)$ is strictly increasing in the initial price $q_{0}$.

2. By consequence, in the case of multiple equilibria (for example, in Proposition 6, Proposition 7, Examples 1-4), the allocation of a bubbly equilibrium strictly Pareto dominates that of the bubbleless equilibrium.

Proof. See Appendix B.3.

Let us provide a sketch of our proof. Recall that the agent i's welfare is $\sum_{t \geq 0} \beta_{i, t} u_{i}\left(c_{i, t}\right)$. By using the FOCs and the concavity of utility functions, we can show that, for any $t$, $\beta_{1,2 t-1} u_{1}\left(c_{1,2 t-1}\right)+\beta_{1,2 t} u_{1}\left(c_{1,2 t}\right)$ and $\beta_{2,2 t} u_{2}\left(c_{2,2 t}\right)+\beta_{2,2 t+1} u_{2}\left(c_{2,2 t+1}\right)$ are strictly positive and strictly increasing in $q_{0}$. By consequence, we can prove that $\sum_{t=0}^{T} \beta_{i, t}\left(u_{i}\left(c_{i, t}\right)-\right.$ $\left.u_{i}\left(c_{i, t}^{\prime}\right)\right)$ converge to a positive number when $T$ tends to infinity, for any two equilibria $\left(q_{t},\left(c_{i, t}\right)_{i}\right)$ and $\left(q_{t}^{\prime},\left(c_{i, t}^{\prime}\right)_{i}\right)$ with $q_{0}>q_{0}^{\prime}$.

To understand the intuition behind our result, let us look at, for example $\beta_{1,2 t-1} u_{1}\left(c_{1,2 t-1}\right)+$ $\beta_{1,2 t} u_{1}\left(c_{1,2 t}\right)$ which equals to

$$
\beta_{1,2 t-1} u_{1}\left(e_{1,2 t-1}-b_{1}^{*} d_{2 t-1}-q_{2 t-1} H\right)+\beta_{1,2 t} u_{1}\left(e_{1,2 t}+d_{2 t}\left(L+b_{2}^{*}\right)+q_{2 t} H\right)
$$

Recall that the rate of substitution $\frac{\beta_{1,2 t} u_{1}^{\prime}\left(e_{1,2 t}\right)}{\beta_{1,2 t-1} u_{1}^{\prime}\left(e_{1,2 t-1}\right)}$ is high (see conditions (13) and (14)) which implies that $e_{1,2 t-1}$ is relatively high with respect to $e_{1,2 t}$. When $q_{0}$ 
increases, both $q_{2 t}$ and $q_{2 t-1}$ increase. This implies that $c_{1,2 t-1}$ decreases and $c_{1,2 t}$ increases, and then helps agent 1 to better smooth her consumption. So, increasing $q_{0}$ is welfare-improving.

Remark 5. Our welfare analysis is consistent with that in Proposition 9 of Hirano and Yanagawa (2017). The different is that we work with general utility functions while they only focus on the logarithmic utility function.

Remark 6. Notice that increasing $q_{0}$ is not necessarily strictly welfare-improving if the utility function $u_{i}$ of some agent $i$ is linear. Indeed, assume that $u_{i}(c)=$ $u_{i} c \forall c$ where $u_{i}>0$. We can check that $W_{i}\left(q_{0}\right)=W_{i}\left(q_{0}^{\prime}\right)$ for any two equilibria satisfying conditions (8) and (9a-9c) (because $\beta_{1,2 t-1} u_{1}\left(c_{1,2 t-1}\right)+\beta_{1,2 t} u_{1}\left(c_{1,2 t}\right)$ and $\beta_{2,2 t} u_{2}\left(c_{2,2 t}\right)+\beta_{2,2 t+1} u_{2}\left(c_{2,2 t+1}\right)$ do not depend on the initial equilibrium price $\left.q_{0}\right) .{ }^{25}$

Remark 7. In a particular case when assets have no dividend, the consumption allocation of the bubbleless equilibrium coincides with that of the autarkic equilibrium. Since the utility function is strictly concave, we can easily prove that $U_{i}\left(c_{i}\right)>U_{i}\left(e_{i}\right)$ $\forall i$. So, its allocation is strictly Pareto dominated by that of bubbly equilibrium. This argument has been used in many papers in the literature. However, it can no longer be applied for the case of positive dividend because the consumption allocation of the bubbleless equilibrium is different from that of the autarkic equilibrium. By the way, our proof of Proposition 9 is new and so part of our contribution.

\section{Conclusion and discussion}

In general equilibrium models with infinitely lived agents, we have provided new conditions (based on fundamentals) under which assets (with or without dividend) do not generate price bubbles. In general, the existence of bubbles is associated with the fluctuations of individual asset trading. However, the existence of bubbles is not a matter of a single factor but the result of an interaction between heterogeneous agents in an imperfect market.

We have provided several mechanisms where bubbles and real indeterminacy exist in equilibrium in a model economy with two kinds of agents. Our analyses suggest that bubbles are more likely to exist if (1) heterogeneity of agents takes place at any period, (2) borrowing limits are tight, (3) the interest rates of the benchmark economy are low so that agents are willing to buy assets even at a high price, (4) there is an asset shortage (the asset supply is low or asset dividends are low to agents' endowments). We have also proved that bubbles may not exist if one of these conditions is violated.

We end our paper by mentioning some avenues of research in the future. First, it would be interesting to understand how our results would be extended in a stochastic economy. Although the existing literature has shown many conditions ruling out bubbles in stochastic general equilibrium models, there are very few examples of bubbles

\footnotetext{
${ }^{25}$ See (B.29) and (B.30) in Appendix.
} 
in such models. To address this question, it is important to understand how agents' decision depends on the economy's fundamentals and the degree of incompleteness of the financial market. Second, are bubbles always welfare-improving? It is true under conditions in Proposition 9. However, since we have not yet found the whole set of equilibria in the general setup, our results are not enough to conclude that bubbles are always welfare-improving. An open question is to investigate whether in a general model any bubbly equilibrium dominates any bubbleless equilibrium.

\section{Acknowledgments}

We thank Tomohiro Hirano and Carmen Camacho for constructive suggestions and comments.

\section{A Equilibrium properties and no-bubble conditions}

Proof of Proposition 1. Part 1. To prove the FOCs, it suffices to prove that: $q_{t} \beta_{i, t} u_{i}^{\prime}\left(c_{i, t}\right) \geq \beta_{i, t+1} u_{i}^{\prime}\left(c_{i, t+1}\right)\left(q_{t+1}+d_{t+1}\right)$ and we have equality if $b_{i, t}+b_{i}^{*}>0$. We can do so by using the standard argument in the dynamic programming (see our working paper Bosi et al. (2019) for instance).

We now prove the TVCs. The FOCs imply that the sequence $\left(\lambda_{i, t} q_{t}\right)_{t}$ is decreasing in $t$. Moreover, we have

$$
\lambda_{i, t} q_{t} b_{i, t}=\left(\lambda_{i, t+1}\left(q_{t+1}+d_{t+1}\right)+\eta_{i, t}\right) b_{i, t}=\lambda_{i, t+1}\left(q_{t+1}+d_{t+1}\right) b_{i, t}-\eta_{i, t} b_{i}^{*}
$$

We rewrite the budget constraint of agent $i$ at date $t$ as follows

$$
\lambda_{i, t}\left(c_{i, t}-e_{i, t}\right)=\lambda_{i, t}\left(q_{t}+d_{t}\right) b_{i, t-1}-\lambda_{i, t} q_{t} b_{i, t}
$$

By taking the sum of this constraint from $t=0$ until $T$ and using (1b), we get

$$
\begin{aligned}
\sum_{t=0}^{T} \lambda_{i, t}\left(c_{i, t}-e_{i, t}\right) & =\sum_{t=0}^{T}\left(\lambda_{i, t}\left(q_{t}+d_{t}\right) b_{i, t-1}-\lambda_{i, t} q_{t} b_{i, t}\right) \\
& =\lambda_{i, 0}\left(q_{0}+d_{0}\right) b_{i,-1}-\lambda_{i, T} q_{T} b_{i, T}+\sum_{t=1}^{T} \eta_{i, t} b_{i}^{*}
\end{aligned}
$$

and hence $\lambda_{i, 0}\left(q_{0}+d_{0}\right) b_{i,-1}+\sum_{t=0}^{T} \lambda_{i, t} e_{i, t}+\sum_{t=1}^{T} \eta_{i, t} b_{i}^{*}=\lambda_{i, T} q_{T} b_{i, T}+\sum_{t=0}^{T} \lambda_{i, t} c_{i, t}$.

We will prove that $\lim _{T \rightarrow+\infty} \lambda_{i, T} q_{T}\left(b_{i, T}+b^{*}\right)$ exists in $\mathbb{R}^{+}$. Recall that the sequence $\left(\lambda_{i, t} q_{t}\right)_{t}$ is positive and decreasing in $t$. So, $\lim _{t \rightarrow+\infty} \lambda_{i, t} q_{t}$ exists and is in $\mathbb{R}^{+}$. We have $-b_{i}^{*} \leq b_{i, t}=L-\sum_{j \neq i} b_{j, t} \leq L+\sum_{i} b_{i}^{*} \forall t$, and hence

$$
-\infty<\liminf _{T \rightarrow+\infty} \lambda_{i, T} q_{T} b_{i, T} \leq \limsup _{T \rightarrow+\infty} \lambda_{i, T} q_{T} b_{i, T}<\infty .
$$


Under our assumptions, we have $\sum_{t} \lambda_{i, t} c_{i, t}<\infty \forall i$. Indeed, we have $\sum_{t} \lambda_{i, t} c_{i, t}=$ $\sum_{t} \beta_{i, t} u_{i}^{\prime}\left(c_{i, t}\right) c_{i, t} \leq \sum_{t} \beta_{i, t} v\left(c_{i, t}\right) \leq \sum_{t} \beta_{i, t} v\left(\sum_{i} e_{i, t}+L d_{t}\right)<\infty$. Thus, we obtain that $\sum_{t} \lambda_{i, t} c_{i, t}<\infty \forall i$

Since $\sum_{t} \lambda_{i, t} c_{i, t}<\infty$, both series $\sum_{t} \lambda_{i, t} e_{i, t}$ and $\sum_{t} \eta_{i, t} b_{i}^{*}$ converge. By consequence, $\lim _{T \rightarrow+\infty} \lambda_{i, T} q_{T} b_{i, T}$ exists in $\mathbb{R}$. Therefore $\lambda_{i, T} q_{T}\left(b_{i, T}+b^{*}\right)$ converges and

$$
\lim _{T \rightarrow+\infty} \lambda_{i, T} q_{T}\left(b_{i, T}+b^{*}\right)=\lim _{T \rightarrow+\infty} \lambda_{i, T} q_{T} b_{i, T}+\lim _{T \rightarrow+\infty} \lambda_{i, T} q_{T} b_{i}^{*} \in \mathbb{R}
$$

There are two cases:

- Case (a): If $\liminf _{t \rightarrow+\infty}\left(b_{i, t}+b_{i}^{*}\right)=0$, then $\lim _{t \rightarrow+\infty} \lambda_{i, t} q_{t}\left(b_{i, t}+b^{*}\right)=0$ because $\lambda_{i, t} q_{t} \leq \lambda_{i, 0} q_{0} \forall t$.

- Case (b): If $\liminf \operatorname{in}_{t \rightarrow+\infty}\left(b_{i, t}+b_{i}^{*}\right)>0$, then there exist $\alpha>0$ and $T$ such that $b_{i, t}+b_{i}^{*}>\alpha \forall t \geq T$. In this case $\eta_{i, t}=0 \forall t \geq T$. For simplicity of the proof, assume $T=0$. We know that $\lim _{t \rightarrow+\infty} \lambda_{i, t} q_{t}$ exists. Let $\zeta \equiv \lim _{t \rightarrow+\infty} \lambda_{i, t} q_{t}$. We claim that $\zeta=0$. Assume the contrary: $\zeta>0$. In this case $\zeta=\lim _{\tau \rightarrow+\infty} \lambda_{i, T+\tau+1} q_{T+\tau+1} \leq \lambda_{i, T} q_{T} \forall T$. Construct a sequence $\left(c_{i, t}^{\prime}, b_{i, t}^{\prime}\right)$ as follows:

$$
c_{i, 0}^{\prime}=c_{i, 0}+\frac{\zeta \alpha}{\lambda_{i, 0}}, c_{i, t}^{\prime}=c_{i, t}, \forall t \geq 1, \quad b_{i, t}^{\prime}=b_{i, t}-\frac{\zeta \alpha}{q_{t} \lambda_{i, t}}, \forall t \geq 0
$$

Since $b_{i, t}^{\prime} \geq-b_{i}^{*}+\alpha-\frac{\zeta \alpha}{q_{t} \lambda_{i, t}}=-b_{i}^{*}+\alpha\left(1-\frac{\zeta}{q_{t} \lambda_{i, t}}\right) \geq-b_{i}^{*}, \forall t$, the sequence $\left(c_{i, t}^{\prime}, b_{i, t}^{\prime}\right)$ satisfies physical, budget and borrowing constraints. However $\sum_{t=0}^{+\infty} \beta_{i, t} u_{i}\left(c_{i, t}^{\prime}\right)>$ $\sum_{t=0}^{+\infty} \beta_{i, t} u_{i}\left(c_{i, t}\right)$ which is a contradiction. Hence $\zeta=0$, i.e. $\lim _{t \rightarrow \infty} q_{t} \lambda_{i, t}=0$. Since $b_{i, t}+b_{i}^{*}=L-\sum_{j \neq i} b_{j, t}+b_{i}^{*} \leq L+\sum_{i} b_{i}^{*} \forall t$, we get

$$
\lambda_{i, t} q_{t}\left(L+\sum_{i} b_{i}^{*}\right) \geq \lambda_{i, t} q_{t}\left(b_{i, t}+b_{t}^{*}\right) \geq \lambda_{i, t} q_{t} \alpha .
$$

This implies that $\lim _{t \rightarrow \infty} \lambda_{i, t} q_{t}\left(b_{i, t}+b_{t}^{*}\right)=0$.

By combining the two cases (a) and (b), we obtain that $\lim _{t \rightarrow \infty} \lambda_{i, t} q_{t}\left(b_{i, t}+b_{t}^{*}\right)=0$.

Part 2 (sufficient condition). It suffices to prove the optimality of the allocation $\left(c_{i}, b_{i}\right)$. Consider another sequence $\left(c_{i}^{\prime}, b_{i}^{\prime}\right)$ satisfying physical, budget and borrowing 
constraints. We have, for any $T$,

$$
\begin{aligned}
\sum_{t=0}^{T} \lambda_{i, t}\left(c_{i, t}-c_{i, t}^{\prime}\right) & \geq \sum_{t=0}^{T} \lambda_{i, t}\left(e_{i, t}+\left(q_{t}+d_{t}\right) b_{i, t-1}-q_{t} b_{i, t}-e_{i, t}-\left(q_{t}+d_{t}\right) b_{i, t-1}^{\prime}+q_{t} b_{i, t}^{\prime}\right) \\
& =\sum_{t=0}^{T-1} \lambda_{i, t+1}\left(q_{t+1}+d_{t+1}\right)\left(b_{i, t}-b_{i, t}^{\prime}\right)-\sum_{t=0}^{T-1} \lambda_{i, t} q_{t}\left(b_{i, t}-b_{i, t}^{\prime}\right)-q_{T} \lambda_{i, T}\left(b_{i, T}-b_{i, T}^{\prime}\right) \\
& =-q_{T} \lambda_{i, T}\left(b_{i, T}-b_{i, T}^{\prime}\right)+\sum_{t=0}^{T-1}\left(\lambda_{i, t+1}\left(q_{t+1}+d_{t+1}\right)-\lambda_{i, t} q_{t}\right)\left(b_{i, t}-b_{i, t}^{\prime}\right) \\
& =-q_{T} \lambda_{i, T}\left(b_{i, T}-b_{i, T}^{\prime}\right)+\sum_{t=0}^{T-1} \eta_{i, t}\left(b_{i, t}^{\prime}-b_{i, t}\right) \\
& =-q_{T} \lambda_{i, T}\left(b_{i, T}+b_{i}^{*}-\left(b_{i, T}^{\prime}+b_{i}^{*}\right)\right)+\sum_{t=0}^{T-1} \eta_{i, t}\left(b_{i, t}^{\prime}+b_{i}^{*}-\left(b_{i, t}+b_{i}^{*}\right)\right) \\
& \geq-q_{T} \lambda_{i, T}\left(b_{i, T}+b_{i}^{*}\right)+\sum_{t=0}^{T-1} \eta_{i, t}\left(b_{i, t}^{\prime}+b_{i}^{*}\right) \geq-q_{T} \lambda_{i, T}\left(b_{i, T}+b_{i}^{*}\right) .
\end{aligned}
$$

Therefore, we have

$$
\sum_{t=0}^{T}\left(\beta_{i, t} u\left(c_{i, t}\right)-\beta_{i, t} u\left(c_{i, t}^{\prime}\right)\right) \geq \sum_{t=0}^{T} \lambda_{i, t}\left(c_{i, t}-c_{i, t}^{\prime}\right) \geq-q_{T} \lambda_{i, T}\left(b_{i, T}+b_{T}^{*}\right) .
$$

Denote $U_{T} \equiv \sum_{t=0}^{T} \beta_{i, t} u\left(c_{i, t}\right)$ and $U_{T}^{\prime} \equiv \sum_{t=0}^{T} \beta_{i, t} u\left(c_{i, t}^{\prime}\right)$. Observe that the sequence $U_{T}$ converges when $T$ tends to infinity.

If $\lim _{T \rightarrow \infty} q_{T} \lambda_{i, T}\left(b_{i, T}+b_{T}^{*}\right)=0$, then $\lim \sup _{T \rightarrow \infty} U_{T}^{\prime} \leq \lim _{T \rightarrow \infty} U_{T}$; we have finished our proof.

Remark 8. If $u_{i}(0) \geq 0$, then the series $\sum_{t=0}^{\infty} \lambda_{i, t} u_{i}\left(c_{i, t}\right)$ always converges. By consequence, conditions $U_{T} \geq U_{T}^{\prime}-q_{T} \lambda_{i, T}\left(b_{i, T}+b_{T}^{*}\right) \forall T$ and $\liminf _{T \rightarrow \infty} q_{T} \lambda_{i, T}\left(b_{i, T}+b_{T}^{*}\right)=0$ imply that $\lim _{T \rightarrow \infty} U_{T} \geq \lim _{T \rightarrow \infty} U_{T}^{\prime}=\lim \sup _{T \rightarrow \infty} U_{T}^{\prime}$.

Proof of Proposition 2. We mainly use Proposition 1.

1. Suppose that there exists $i$ such that $\liminf _{t \rightarrow \infty}\left(b_{i, t}+b_{i}^{*}\right)>0$. In this case, there exists $T$ such that $b_{i, t}+b_{i}^{*}>0 \forall t \geq T$. So, $\frac{\lambda_{i, t+1}}{\lambda_{i, t}}=\frac{q_{t}}{q_{t+1}+d_{t+1}}=\frac{1}{R_{t+1}} \forall t \geq T$. This implies that $Q_{t}=Q_{T} \frac{\lambda_{i, t}}{\lambda_{i, T}} \forall t \geq T$. By combining with the TVC, we get that $\lim _{t \rightarrow \infty} Q_{t} q_{t}\left(b_{i, t}+b_{i}^{*}\right)=0 \forall i$. This is impossible because $\liminf _{t \rightarrow \infty}\left(b_{i, t}+b_{i}^{*}\right)>0$ and $\lim _{\rightarrow \infty} Q_{t} q_{t}>0$.

2. We firstly prove that: there exist an agent, say agent $i$, and an infinite, increasing sequence $\left(i_{n}\right)_{n}$ such that $b_{i, i_{n}}+b_{i}^{*}=0$ for all $n=0,1, \ldots$. Indeed, assume, 
by contradiction, that for any agent $i$, there exists $T$ such that $b_{i, t}>-b_{i}^{*} \forall t \geq T$. As discussed above, we obtain that $\lim _{t \rightarrow \infty} Q_{t} q_{t}\left(b_{i, t}+b_{i}^{*}\right)=0 \forall i$. Taking the sum over $i$ and using market clearing conditions, we get that $\lim _{t \rightarrow \infty} Q_{t} q_{t}=0$, i.e., there is no bubble, a contradiction.

We now consider other agents $j \in\{2, \cdots, m\}$. Suppose that for any $j \geq 2$, there is $T_{j}$ such that $b_{j, t}+b_{j}^{*}>0 \forall t \geq T_{j}$. So, $\frac{\lambda_{j, t+1}}{\lambda_{j, t}}=\frac{q_{t}}{q_{t+1}+d_{t+1}}=\frac{1}{R_{t+1}} \forall j \geq 2, \forall t \geq$ $T \equiv \max _{j \geq 2} T_{j}$, which implies that $Q_{t}=Q_{T} \frac{\lambda_{j, t}}{\lambda_{j, T}} \forall j \geq 2, t \geq T$. By combining with the TVC, we get that $\lim _{t \rightarrow \infty} Q_{t} q_{t}\left(b_{j, t}+b_{j}^{*}\right)=0$. Since bubbles exist, we have $\lim _{t \rightarrow \infty} Q_{t} q_{t}>0$. We then get that $\lim _{t \rightarrow \infty}\left(b_{j, t}+b_{j}^{*}\right)=0$. Market clearing conditions imply that $\lim _{t \rightarrow \infty} b_{1, t}+b_{1}^{*}=L-\lim _{t \rightarrow \infty} \sum_{j \geq 2} b_{j, t}+b_{1}^{*}=L+\sum_{i=1}^{m} b_{i}^{*}>0$. So, there exists $T_{1}$ such that $b_{1, t}+b_{1}^{*}>0 \forall t \geq T_{1}$, which is a contradiction. By consequence, there exist an agent, say agent 2 , and an increasing sequence $\left(j_{n}\right)_{n}$ such that $b_{j, j_{n}}+b_{j}^{*}=$ 0 for all $n=0,1, \ldots$

3. Suppose that there exist $m-1$ agents such that their asset holding converges. By market clearing conditions, the asset holding of all agents converges. So, there exists an agent $i$ such that $\lim _{t \rightarrow \infty} b_{i, t}>0$. According to point 1 , this is impossible.

Proof of Corollary 2. Suppose that $\sum_{t>0} Q_{t} e_{i, t}<\infty \forall i$. Budget constraint of agent $i$ implies that $Q_{t} c_{i, t}+Q_{t} q_{t} b_{i, t}=Q_{t} e_{i, t}+Q_{t}\left(q_{t}+d_{t}\right) b_{i, t-1}$. By summing this equation over $t$ and noticing that $Q_{t} q_{t}=Q_{t+1}\left(q_{t+1}+d_{t+1}\right)$, we have

$$
\sum_{t=0}^{T} Q_{t} c_{i, t}+Q_{T} q_{T} b_{i, T}=\sum_{t=0}^{T} Q_{t} e_{i, t}+\left(q_{0}+d_{0}\right) b_{i,-1} \forall t .
$$

Since $\sum_{t>0} Q_{t} e_{i, t}<\infty$ and $\left(Q_{T} q_{T} b_{i, T}\right)$ is bounded (because $b_{i, T}$ and $Q_{T} q_{T}$ are bounded), the series $\sum_{t>0} Q_{t} c_{i, t}$ converges, and so does the sequence $\left(Q_{T} q_{T} b_{i, T}\right)_{T}$. If there is a bubble, we have $\lim _{t \rightarrow \infty} Q_{t} q_{t}>0$. By consequence, $\left(b_{i, t}\right)$ converges for any $i$. Market clearing conditions imply that there is an agent $i$ such that $b_{i} \equiv \lim _{t \rightarrow \infty} b_{i, t}>0$. So, borrowing constraints of agent $i$ do not bind from some date on, say $T$. Hence, $\frac{\lambda_{i, t+1}}{\lambda_{i, t}}=\frac{q_{t}}{q_{t+1}+d_{t+1}}=\frac{1}{R_{t+1}} \forall t \geq T$. This implies that $Q_{t}=Q_{T} \frac{\lambda_{i, t}}{\lambda_{i, T}} \forall t \geq T$. By combining with the TVC, we get that $\lim _{t \rightarrow \infty} Q_{t} q_{t}\left(b_{i, t}+b_{i}^{*}\right)=0 \forall i$. This is impossible because $\lim _{t \rightarrow \infty}\left(b_{i, t}+b_{i}^{*}\right)>0$ and $\lim _{t} Q_{t} q_{t}>0$.

If $\liminf \inf _{t} \frac{d_{t}}{\sum_{i} e_{i, t}}>0$, there exist a date $t_{0} \geq 1$ and a positive constant $x$ such that $x d_{t} \geq \sum_{i} e_{i, t} \forall t \geq t_{0}$. Therefore, we have

$$
\begin{aligned}
\sum_{t \geq 0} Q_{t}\left(\sum_{i} e_{i, t}\right) & =\sum_{t=0}^{t_{0}-1} Q_{t}\left(\sum_{i} e_{i, t}\right)+\sum_{t \geq t_{0}} Q_{t}\left(\sum_{i} e_{i, t}\right) \\
& \leq \sum_{t=0}^{t_{0}-1} Q_{t}\left(\sum_{i} e_{i, t}\right)+x \sum_{t \geq t_{0}} Q_{t} d_{t} \leq \sum_{t=0}^{t_{0}-1} Q_{t}\left(\sum_{i} e_{i, t}\right)+x q_{0}<\infty .
\end{aligned}
$$

Hence $\sum_{t \geq 0} Q_{t}\left(\sum_{i} e_{i, t}\right)<\infty$, and so there does not exist bubble. 
Proof of Proposition 3. We need intermediate results (Lemmas 1, 2, 3).

Lemma 1. At each date $t$, there exists $i$ such that $b_{i, t} \geq b_{i, t+1}$ and borrowing constraint is not binding (i.e., $b_{i, t}+b_{i}^{*}>0$ ).

Proof. Define $i_{0}$ such that $b_{i_{0}, t}-b_{i_{0}, t+1}=\max _{i}\left\{b_{i, t}-b_{i, t+1}\right\}$. Then, we have $b_{i_{0}, t}-$ $b_{i_{0}, t+1} \geq 0$. We consider two cases.

Case 1: If $b_{i_{0}, t}-b_{i_{0}, t+1}>0$, then $b_{i_{0}, t}+b_{i}^{*}>b_{i_{0}, t+1}+b_{i}^{*} \geq 0$.

Case 2: If $b_{i_{0}, t}-b_{i_{0}, t+1}=0$, then $b_{i, t}-b_{i, t+1} \leq 0 \forall i$. Since $\sum_{i}\left(b_{i, t}-b_{i, t+1}\right)=0$, we get that $b_{i, t}-b_{i, t+1}=0$ for every $i$. Since $\sum_{i} b_{i, t}>0$, we can choose $i_{1}$ such that $b_{i_{1}, t}>0$. So, we have $b_{i_{1}, t}=b_{i_{1}, t+1}$ and $b_{i_{1}, t}+b_{i}^{*}>0$.

Lemma 2. Assume that $e_{i, t}-d_{t} b_{i}^{*}>0 \forall i, \forall t$, then we have

$$
\frac{1}{R_{t+1}} \leq \max _{i} \frac{\beta_{i, t+1} u_{i}^{\prime}\left(e_{i, t+1}-d_{t+1} b_{i}^{*}\right)}{\beta_{i, t} u_{i}^{\prime}\left(\sum_{i} e_{i, t}+L d_{t}\right)} \forall t \geq 0 .
$$

Proof. Recall that we have $1=R_{t+1} \max _{i} \frac{\beta_{i, t+1} u_{i}^{\prime}\left(c_{i, t+1}\right)}{\beta_{i, t} u_{i}^{\prime}\left(c_{i, t}\right)} \forall t \geq 0$. Let $t \geq 0$, Lemma 1 implies that there exists an agent $i=i(t)$ (depending on $t$ ) such that $b_{i(t), t} \geq b_{i(t), t+1}$ and $b_{i(t), t}+b_{i(t)}^{*}>0$. Then, we have $\eta_{i(t), t}=0$ and hence

$$
1=R_{t+1} \frac{\beta_{i(t), t+1} u_{i(t)}^{\prime}\left(c_{i(t), t+1}\right)}{\beta_{i(t), t} u_{i(t)}^{\prime}\left(c_{i(t), t}\right)} .
$$

We observe that $c_{i(t), t+1}=e_{i(t), t+1}+\left(q_{t+1}+d_{t+1}\right) b_{i(t), t}-q_{t+1} b_{i(t), t+1} \geq e_{i(t), t+1}-$ $d_{t+1} b_{i(t)}^{*}$ and $c_{i(t), t} \leq W_{t} \equiv \sum_{i} e_{i, t}+L d_{t}$. By consequence, we get that

$\frac{1}{R_{t+1}}=\frac{\beta_{i(t), t+1} u_{i(t)}^{\prime}\left(c_{i(t), t+1}\right)}{\beta_{i(t), t} u_{i(t)}^{\prime}\left(c_{i(t), t}\right)} \leq \frac{\beta_{i(t), t+1} u_{i(t)}^{\prime}\left(e_{i(t), t+1}-d_{t+1} b_{i(t)}^{*}\right)}{\beta_{i(t), t} u_{i(t)}^{\prime}\left(\sum_{i} e_{i, t}+L d_{t}\right)} \leq \max _{i} \frac{\beta_{i, t+1} u_{i}^{\prime}\left(e_{i, t+1}-d_{t+1} b_{i}^{*}\right)}{\beta_{i, t} u_{i}^{\prime}\left(\sum_{i} e_{i, t}+L d_{t}\right)}$

Lemma 3. Consider an equilibrium. Take $\gamma$ in Assumption 3, we have that (1$\gamma) q_{t} b_{i, t} \leq W_{t} \forall i, \forall t$.

Proof. Suppose, by contradiction, that there exist $i$ and $t$ such that $(1-\gamma) q_{t} b_{i, t}>W_{t}$. Let us consider a new allocation of agent $i: z_{i}:=z\left(c_{i}, t, \gamma,(1-\gamma) q_{t} b_{i, t}\right)$. We check that this allocation is in the budget set of agent $i$ because

$$
\begin{array}{r}
\left(c_{i, t}+(1-\gamma) q_{t} b_{i, t}\right)+q_{t}\left(\gamma b_{i, t}\right) \leq e_{i, t}+\left(q_{t}+d_{t}\right) b_{i, t-1} \\
\gamma c_{i, s}+q_{s}\left(\gamma b_{i, s}\right)=\gamma e_{i, s}+\left(q_{t}+d_{t}\right)\left(\gamma b_{i, s-1}\right) \leq e_{i, s}+\left(q_{t}+d_{t}\right)\left(\gamma b_{i, s-1}\right) \forall s \geq t+1
\end{array}
$$

According to Assumption 3, we have

$$
U_{i}\left(c_{i}\right)<U_{i}\left(z\left(c_{i}, t, \gamma, W_{t}\right)<U_{i}\left(z\left(c_{i}, t, \gamma,(1-\gamma) q_{t} b_{i, t}\right)\right) .\right.
$$

This is in contradiction to the optimality of $\left(c_{i}, b_{i}\right)$. 
We now prove Proposition 3. Since points 1 and 2 are direct consequences of (4), let us prove (4). According to Lemma 3, we have $(1-\gamma) q_{t} b_{i, t} \leq W_{t} \forall i, \forall t$. Taking the sum over $i$, we get $(1-\gamma) q_{t} L \leq m W_{t} \forall t$. Since $L(1-\gamma)>0$, we get that

$$
q_{t} \leq \frac{m W_{t}}{L(1-\gamma)} \forall t
$$

According to Lemma 2, we have

$$
\frac{1}{R_{t+1}} \leq \max _{i} \frac{\beta_{i, t+1} u_{i}^{\prime}\left(e_{i, t+1}-d_{t+1} b_{i}^{*}\right)}{\beta_{i, t} u_{i}^{\prime}\left(\sum_{i} e_{i, t}+L d_{t}\right)} \forall t \geq 0 .
$$

Recall that there is no bubble iff $\lim _{t \rightarrow \infty} Q_{t} q_{t}=0$. By combining these above arguments, there is no bubble if condition (4) is satisfied.

\section{B Proofs for Section 4. Models with bubbles}

We firstly state and prove a condition under which a sequence is a system of prices. This condition is very useful for the next proofs.

Lemma 4. Let Assumption 1, 2 be satisfied.

(1) The sequence $\left(q_{t}\right)_{t \geq 0}$, asset holdings given by (8) and agents' consumptions given by (9a-9c) constitute an equilibrium if and only if consumptions are strictly positive and the following conditions hold

$$
\begin{aligned}
& 1=\gamma_{2,0} \frac{u_{2}^{\prime}\left(e_{2,1}+d_{1}\left(L+b_{1}^{*}\right)+q_{1} H\right)}{u_{2}^{\prime}\left(e_{2,0}+\left(q_{0}+d_{0}\right) b_{2,-1}-q_{0}\left(L+b_{1}^{*}\right)\right)} \frac{q_{1}+d_{1}}{q_{0}} \\
& 1=\gamma_{1,2 t-1} \frac{u_{1}^{\prime}\left(e_{1,2 t}+d_{2 t}\left(L+b_{2}^{*}\right)+q_{2 t} H\right)}{u_{1}^{\prime}\left(e_{1,2 t-1}-b_{1}^{*} d_{2 t-1}-q_{2 t-1} H\right)} \frac{q_{2 t}+d_{2 t}}{q_{2 t-1}} \\
& 1=\gamma_{2,2 t} \frac{u_{2}^{\prime}\left(e_{2,2 t+1}+d_{2 t+1}\left(L+b_{1}^{*}\right)+q_{2 t+1} H\right)}{u_{2}^{\prime}\left(e_{2,2 t}-b_{2}^{*} d_{2 t}-q_{2 t} H\right)} \frac{q_{2 t+1}+d_{2 t+1}}{q_{2 t}} \\
& \gamma_{1,2 t-1} \frac{u_{1}^{\prime}\left(e_{1,2 t}+d_{2 t}\left(L+b_{2}^{*}\right)+q_{2 t} H\right)}{u_{1}^{\prime}\left(e_{1,2 t-1}-b_{1}^{*} d_{2 t-1}-q_{2 t-1} H\right)} \geq \frac{\gamma_{2,2 t-1} u_{2}^{\prime}\left(e_{2,2 t}-d_{2 t} b_{2}^{*}-q_{2 t} H\right)}{u_{2}^{\prime}\left(e_{2,2 t-1}+d_{2 t-1}\left(L+b_{1}^{*}\right)+q_{2 t-1} H\right)} \\
& \gamma_{2,2 t} \frac{u_{2}^{\prime}\left(e_{2,2 t+1}+d_{2 t+1}\left(L+b_{1}^{*}\right)+q_{2 t+1} H\right)}{u_{2}^{\prime}\left(e_{2,2 t}-d_{2 t} b_{2}^{*}-q_{2 t} H\right)} \geq \gamma_{1,2 t} \frac{u_{1}^{\prime}\left(e_{1,2 t+1}-b_{1}^{*} d_{2 t+1}-q_{2 t+1} H\right)}{u_{1}^{\prime}\left(e_{1,2 t}+d_{2 t}\left(L+b_{2}^{*}\right)+q_{2 t} H\right)} \\
& \gamma_{2,0} \frac{u_{2}^{\prime}\left(e_{2,1}+d_{1}\left(L+b_{1}^{*}\right)+q_{1} H\right)}{u_{2}^{\prime}\left(e_{2,0}+\left(q_{0}+d_{0}\right) b_{2,-1}-q_{0}\left(L+b_{1}^{*}\right)\right)} \geq \gamma_{1,0} \frac{u_{1}^{\prime}\left(e_{1,1}-b_{1}^{*} d_{1}-q_{1} H\right)}{u_{1}^{\prime}\left(e_{1,0}+\left(q_{0}+d_{0}\right) b_{1,-1}+q_{0} b_{1}^{*}\right)}
\end{aligned}
$$


and

$$
\begin{array}{r}
\lim _{t \rightarrow \infty} \beta_{1,2 t+1} u_{1}^{\prime}\left(e_{1,2 t+1}-b_{1}^{*} d_{2 t+1}-q_{2 t+1} H\right) q_{2 t+1} H=0 \\
\lim _{t \rightarrow \infty} \beta_{2,2 t} u_{2}^{\prime}\left(e_{2,2 t}-d_{2 t} b_{2}^{*}-q_{2 t} H\right) q_{2 t} H=0
\end{array}
$$

Observe that the TVCs hold if $\lim _{t \rightarrow \infty} \beta_{2,2 t} u_{2}^{\prime}\left(e_{2,2 t}\right) e_{2,2 t}=\lim _{t \rightarrow \infty} \beta_{1,2 t+1} u_{1}^{\prime}\left(e_{1,2 t+1}\right) e_{1,2 t+1}=0$.

(2) When $u_{i}(c)=\ln (c) \forall i=1,2$, the sequence $\left(q_{t}\right)_{t}$, asset holdings given by (8) and agents' consumptions given by (9a-9c) constitute an equilibrium if, for any $t \geq 1$, $\gamma_{1,2 t-1} \geq \gamma_{2,2 t-1}, \gamma_{2,2 t} \geq \gamma_{1,2 t}$

$$
\begin{aligned}
e_{1,2 t-1} & \geq e_{2,2 t-1}+\left(L+2 b_{1}^{*}\right) d_{2 t-1}+2 H q_{2 t-1} \\
e_{2,2 t} & \geq e_{1,2 t}+\left(L+2 b_{2}^{*}\right) d_{2 t}+2 H q_{2 t} \\
e_{2,0} & \geq e_{1,0}+d_{0}\left(b_{1,-1}-b_{2,-1}\right)+q_{0}\left(L+2 b_{1}^{*}+b_{1,-1}-b_{2,-1}\right)
\end{aligned}
$$

and

$$
\begin{aligned}
q_{0} & =\left(q_{1}+d_{1}\right) \frac{\gamma_{2,0}\left(e_{2,0}+\left(q_{0}+d_{0}\right) b_{2,-1}-q_{0} b_{2,0}\right)}{e_{2,1}+d_{1}\left(L+b_{1}^{*}\right)+q_{1} H} \\
q_{2 t-1} & =\left(q_{2 t}+d_{2 t}\right) \frac{\gamma_{1,2 t-1}\left(e_{1,2 t-1}-b_{1}^{*} d_{2 t-1}-q_{2 t-1} H\right)}{e_{1,2 t}+d_{2 t}\left(L+b_{2}^{*}\right)+q_{2 t} H} \\
q_{2 t} & =\left(q_{2 t+1}+d_{2 t+1}\right) \frac{\gamma_{2,2 t}\left(e_{2,2 t}-b_{2}^{*} d_{2 t}-q_{2 t} H\right)}{e_{2,2 t+1}+d_{2 t+1}\left(L+b_{1}^{*}\right)+q_{2 t+1} H}
\end{aligned}
$$

Proof of Lemma 4. The part 1 of Lemma 4 is a direct consequence of Proposition 1. Let us prove the part 2. Since $u_{i}(c)=\ln (c) \forall i$, we have $u_{i}^{\prime}(c)=1 / c \forall i$, and hence FOCs now become

$$
\begin{aligned}
& 1=\frac{\gamma_{1,2 t-1} u_{1}^{\prime}\left(c_{1,2 t}\right)}{u_{1}^{\prime}\left(c_{1,2 t-1}\right)} \frac{q_{2 t}+d_{2 t}}{q_{2 t-1}}=\frac{\gamma_{1,2 t-1}\left(e_{1,2 t-1}-b_{1}^{*} d_{2 t-1}-q_{2 t-1} H\right)}{e_{1,2 t}+d_{2 t}\left(L+b_{2}^{*}\right)+q_{2 t} H} \frac{q_{2 t}+d_{2 t}}{q_{2 t-1}} \\
& 1=\frac{\gamma_{2,2 t} u_{2}^{\prime}\left(c_{2,2 t+1}\right)}{u_{2}^{\prime}\left(c_{2,2 t}\right)} \frac{q_{2 t+1}+d_{2 t+1}}{q_{2 t}}=\frac{\gamma_{2,2 t}\left(e_{2,2 t}-d_{2 t} b_{2}^{*}-q_{2 t} H\right)}{e_{2,2 t+1}+d_{2 t+1}\left(L+b_{1}^{*}\right)+q_{2 t+1} H} \frac{q_{2 t+1}+d_{2 t+1}}{q_{2 t}} \\
& \frac{\gamma_{1,2 t-1} u_{1}^{\prime}\left(c_{1,2 t}\right)}{u_{1}^{\prime}\left(c_{1,2 t-1}\right)} \geq \frac{\gamma_{2,2 t-1} u_{2}^{\prime}\left(c_{2,2 t}\right)}{u_{2}^{\prime}\left(c_{2,2 t-1}\right)}, \quad \frac{\gamma_{2,2 t} u_{2}^{\prime}\left(c_{2,2 t+1}\right)}{u_{2}^{\prime}\left(c_{2,2 t}\right)} \geq \frac{\gamma_{1,2 t} u_{1}^{\prime}\left(c_{1,2 t+1}\right)}{u_{1}^{\prime}\left(c_{1,2 t}\right)}
\end{aligned}
$$

The two last inequalities of the FOCs become

$$
\begin{aligned}
& \gamma_{1,2 t-1} \frac{e_{1,2 t-1}-b_{1}^{*} d_{2 t-1}-q_{2 t-1} H}{e_{1,2 t}+d_{2 t}\left(L+b_{2}^{*}\right)+q_{2 t} H} \geq \gamma_{2,2 t-1} \frac{e_{2,2 t-1}+d_{2 t-1}\left(L+b_{1}^{*}\right)+q_{2 t-1} H}{e_{2,2 t}-d_{2 t} b_{2}^{*}-q_{2 t} H} \\
& \gamma_{2,2 t} \frac{e_{2,2 t}-d_{2 t} b_{2}^{*}-q_{2 t} H}{e_{2,2 t+1}+d_{2 t+1}\left(L+b_{1}^{*}\right)+q_{2 t+1} H} \geq \gamma_{1,2 t} \frac{e_{1,2 t}+d_{2 t}\left(L+b_{2}^{*}\right)+q_{2 t} H}{e_{1,2 t+1}-b_{1}^{*} d_{2 t+1}-q_{2 t+1} H}
\end{aligned}
$$


At the first period, FOCs are

$$
\begin{gathered}
\frac{\gamma_{2,0} u_{2}^{\prime}\left(c_{2,1}\right)}{u_{2}^{\prime}\left(c_{2,0}\right)} \geq \frac{\gamma_{1,0} u_{1}^{\prime}\left(c_{1,1}\right)}{u_{1}^{\prime}\left(c_{1,0}\right)} \Leftrightarrow \gamma_{2,0} \frac{c_{2,0}}{c_{2,1}} \geq \gamma_{1,0} \frac{c_{1,0}}{c_{1,1}} \\
\Leftrightarrow \gamma_{2,0} \frac{e_{2,0}+\left(q_{0}+d_{0}\right) b_{2,-1}-q_{0} b_{2,0}}{e_{2,1}+d_{1}\left(L+b_{1}^{*}\right)+q_{1} H} \geq \gamma_{1,0} \frac{e_{1,0}+\left(q_{0}+d_{0}\right) b_{1,-1}-q_{0} b_{1,0}}{e_{1,1}-b_{1}^{*} d_{1}-q_{1} H} \\
\text { and } 1=\frac{\gamma_{2,0} u_{2}^{\prime}\left(c_{2,1}\right)}{u_{2}^{\prime}\left(c_{2,0}\right)} \frac{q_{1}+d_{1}}{q_{0}}=\gamma_{2,0} \frac{e_{2,0}+\left(q_{0}+d_{0}\right) b_{2,-1}-q_{0} b_{2,0}}{e_{2,1}+d_{1}\left(L+b_{1}^{*}\right)+q_{1} H} \frac{q_{1}+d_{1}}{q_{0}}
\end{gathered}
$$

These inequalities are guaranteed by conditions (B.4a-B.4c) while these equalities are ensured by conditions (B.5a-B.5c).

According to Proposition 1, it remains to prove the transversality conditions:

$$
\begin{array}{ll}
\lim _{t \rightarrow \infty} q_{2 t}\left(b_{1,2 t}+b_{1}^{*}\right) \lambda_{1,2 t}=0, & \lim _{t \rightarrow \infty} q_{2 t+1}\left(b_{1,2 t+1}+b_{1}^{*}\right) \lambda_{1,2 t+1}=0 \\
\lim _{t \rightarrow \infty} q_{2 t}\left(b_{2,2 t}+b_{2}^{*}\right) \lambda_{2,2 t}=0, & \lim _{t \rightarrow \infty} q_{2 t+1}\left(b_{2,2 t+1}+b_{2}^{*}\right) \lambda_{2,2 t+1}=0 .
\end{array}
$$

Since $b_{1,2 t}=-b_{1}^{*}$ and $b_{2,2 t-1}=-b_{2}^{*}$, they becomes

$$
\lim _{t \rightarrow \infty} q_{2 t+1}\left(b_{1,2 t+1}+b_{1}^{*}\right) \lambda_{1,2 t+1}=0 \text { and } \lim _{t \rightarrow \infty} q_{2 t}\left(b_{2,2 t}+b_{2}^{*}\right) \lambda_{2,2 t}=0
$$

or equivalently, $\lim _{t \rightarrow \infty} q_{2 t+1} H \beta_{1,2 t+1} \frac{1}{c_{1,2 t+1}}=0$ and $\lim _{t \rightarrow \infty} q_{2 t} H \beta_{2,2 t} \frac{1}{c_{2,2 t}}=0$

These conditions are satisfied thank to (B.4a-B.4c).

Proof of Proposition 5. First, we observe that $u_{1}^{\prime}\left(e_{1,2 t}+d_{2 t}\left(L+b_{2}^{*}\right)+q_{2 t} H\right)=$ $u_{1}^{\prime}\left(e_{1,2 t}-d_{2 t} b_{1}^{*}+\left(q_{2 t}+d_{2 t}\right) H\right)$ and $u_{2}^{\prime}\left(e_{2,2 t+1}+d_{2 t+1}\left(L+b_{1}^{*}\right)+q_{2 t+1} H\right)=u_{2}^{\prime}\left(e_{2,2 t+1}-\right.$ $\left.d_{2 t+1} b_{1}^{*}+\left(q_{2 t+1}+d_{2 t+1}\right) H\right)$. Since $e_{1, t}-d_{t} b_{1}^{*}, e_{2, t}-d_{t} b_{2}^{*}$ are strictly positive and the function $c u_{i}^{\prime}(c)$ is increasing in $c$, the three numerators in FOCs (B.1a), (B.1b), (B.1c) are increasing in $q_{1}, q_{2 t}, q_{2 t+1}$ respectively. Moreover, the three denominators in FOCs (B.1a), (B.1b), (B.1c) are increasing in $q_{0}, q_{2 t-1}, q_{2 t}$ respectively. By consequence, $q_{t+1}$ is increasing in $q_{t}$ for any $t$. This in turns implies that $\frac{q_{t+1}+d_{t+1}}{q_{t}}$ is increasing in $q_{t}$ for any $t$. By consequence, $q_{t}$ is strictly increasing in $q_{0}$ and $\frac{1}{R_{t}}=\frac{q_{t-1}}{q_{t}+d_{t}}$ is strictly decreasing in $q_{0}$. Thus, the fundamental value $F V_{0}=\sum_{t \geq 1} Q_{t} d_{t}$ is strictly decreasing in $q_{0}$. Hence, the asset price bubble component $B_{0} \equiv q_{0}-F V_{0}$ is strictly increasing in $q_{0}$.

Second, we prove that $\mathcal{S}_{0}$ is connected. Let $x, y \in \mathcal{S}_{0}$ with $x<y$, and let $z \in(x, y)$. Since $q_{t}(\cdot)$ is a strictly increasing function, we have $q_{t}(y)>q_{t}(z)>q_{t}(x)$. So, the sequence $\left(q_{t}(z)\right)_{t}$ is strictly positive and individual consumptions generated by this sequence are strictly positive. To prove that the sequence $\left(q_{t}(z)\right)$ is a sequence of equilibrium prices, we have to now verify FOCs and TVCs. FOCs (B.1a), (B.1b), 
(B.1c) are obviously satisfied. We present a proof of (B.1d) (conditions (B.1e), (B.1f) can be proved by applying the same method). Indeed, we have

$$
\begin{aligned}
\frac{\gamma_{1,2 t-1} u_{1}^{\prime}\left(e_{1,2 t}+d_{2 t}\left(L+b_{2}^{*}\right)+q_{2 t}(z) H\right)}{\gamma_{2,2 t-1} u_{2}^{\prime}\left(e_{2,2 t}-d_{2 t} b_{2}^{*}-q_{2 t}(z) H\right)} & \geq \frac{\gamma_{1,2 t-1} u_{1}^{\prime}\left(e_{1,2 t}+d_{2 t}\left(L+b_{2}^{*}\right)+q_{2 t}(y) H\right)}{\gamma_{2,2 t-1} u_{2}^{\prime}\left(e_{2,2 t}-d_{2 t} b_{2}^{*}-q_{2 t}(y) H\right)} \\
& \geq \frac{u_{1}^{\prime}\left(e_{1,2 t-1}-b_{1}^{*} d_{2 t-1}-q_{2 t-1}(y) H\right)}{u_{2}^{\prime}\left(e_{2,2 t-1}+d_{2 t-1}\left(L+b_{1}^{*}\right)+q_{2 t-1}(y) H\right)} \\
& \geq \frac{u_{1}^{\prime}\left(e_{1,2 t-1}-b_{1}^{*} d_{2 t-1}-q_{2 t-1}(z) H\right)}{u_{2}^{\prime}\left(e_{2,2 t-1}+d_{2 t-1}\left(L+b_{1}^{*}\right)+q_{2 t-1}(z) H\right)} .
\end{aligned}
$$

because $q_{t}(y)>q_{t}(z) \forall t$. So, we obtain (B.1d).

TVCs (B.2), (B.3) are satisfied because $q_{t}(y)>q_{t}(z) \forall t, u_{1}^{\prime}\left(e_{1,2 t+1}-b_{1}^{*} d_{2 t+1}-\right.$ $\left.q_{2 t+1} H\right) q_{2 t+1}$ is increasing in $q_{2 t+1}$, and $u_{2}^{\prime}\left(e_{2,2 t}-d_{2 t} b_{2}^{*}-q_{2 t} H\right) q_{2 t}$ is increasing in $q_{2 t}$.

The two last points of Proposition 5 are a direct consequence of the fact that $B_{0} \equiv q_{0}-F V_{0}$ is strictly increasing in $q_{0}$.

\section{B.1 Proofs for Section 4.3.1}

Proof of Example 1. Assume that there exists a bubble, then we have $q_{t}>0 \forall t$. According to FOCs (B.5a-B.5c) we obtain that $\frac{1}{H q_{t+1}}=\frac{\beta e}{w} \frac{1}{H q_{t}}-\frac{1+\beta}{w} \forall t \geq 0$. From this, by iterating, we get that

$$
\frac{1}{H q_{t}}=\frac{1}{H q_{0}} \Gamma_{t}-D_{t}=\frac{1}{H q_{0}}\left(\frac{\beta e}{w}\right)^{t}-\frac{1+\beta}{w}\left(1+\frac{\beta e}{w}+\cdots+\left(\frac{\beta e}{w}\right)^{t-1}\right) \forall t \geq 1
$$

1. If $\frac{\beta e}{w} \leq 1$ (i.e., $R^{*} \geq 1$ ), then the right hand side of (B.11) is negative if $t$ is high enough while the left hand side is strictly positive, a contradiction. Therefore, there is no bubble in this case.

2. If $\frac{\beta e}{w}>1$ (i.e., $R^{*}<1$ ). In this case, we have

$$
\frac{1}{H q_{t}}=\frac{\left(\frac{\beta e}{w}\right)^{t}}{H q_{0}}-\frac{1+\beta}{w} \frac{\left(\frac{\beta e}{w}\right)^{t}-1}{\frac{\beta e}{w}-1}=\frac{\left(\frac{\beta e}{w}\right)^{t}}{H q_{0}}\left(1-H q_{0} \frac{1+\beta}{\beta e-w}\left(1-\left(\frac{w}{\beta e}\right)^{t}\right)\right) \forall t \geq 1
$$

(a) If $q_{0}>\frac{1}{H} \frac{\beta e-w}{1+\beta}$, then $1-H q_{0} \frac{1+\beta}{\beta e-w}<0$. By consequence, the right hand side is strictly negative when $t$ is high enough, a contradiction. In this case, there is no bubble.

(b) If $q_{0}=\frac{1}{H} \frac{\beta e-w}{1+\beta}$, then $1-H q_{0} \frac{1+\beta}{\beta e-w}=0$. By consequence, we have $q_{t}=q>0$ $\forall t \geq 1$. To verify that this is an equilibrium price, we must check conditions (B.4a-B.4c) which now become $e-w>2 H \frac{1}{H} \frac{\beta e-w}{1+\beta}$. This is satisfied because $\beta \in(0,1)$. 
(c) If $0<q_{0}<\frac{1}{H} \frac{\beta e-w}{1+\beta}$, then $1-H q_{0} \frac{1+\beta}{\gamma e-w}>0$. In this case, we see that the sequence $q_{t}$ determined by $(B .12)$ is positive and decreasing in $t$, and $\lim _{t \rightarrow \infty} q_{t}=0$. Conditions (B.4a-B.4c) which now become $e-w>2 H q_{t}$ $\forall t$. Since $q_{0}<\frac{1}{H} \frac{\beta e-w}{1+\beta}$, we have $2 H q_{t} \leq 2 H q_{0}<e-w \forall t$. So, conditions (B.4a-B.4c) are satisfied. Therefore, the sequence $\left(q_{t}\right)_{t}$, determined by two conditions $q_{0}<\frac{1}{H} \frac{\beta e-w}{1+\beta}$ and (B.11), constitutes a system of equilibrium price with bubble.

Proof of Proposition 6. Part 1. Bubble exists iff $q_{t}>0 \forall t$. FOCs (B.5a-B.5c) now become

$$
\left\{\begin{array} { l } 
{ 1 = \gamma _ { 2 , 0 } \frac { e _ { 2 , 0 } + q _ { 0 } b _ { 2 , - 1 } - q _ { 0 } ( L + b _ { 1 } ^ { * } ) } { e _ { 2 , 1 } + q _ { 1 } H } \frac { q _ { 1 } } { q _ { 0 } } } \\
{ 1 = \frac { \gamma _ { t } ( e _ { t } - H q _ { t } ) } { w _ { t + 1 } + H q _ { t + 1 } } \frac { q _ { t + 1 } } { q _ { t } } \forall t \geq 1 }
\end{array} \Leftrightarrow \left\{\begin{array}{ll}
\frac{e_{2,1}}{q_{1}}+H & =\gamma_{2,0} \frac{e_{2,0}}{q_{0}}-\gamma_{2,0}\left(L+b_{1}^{*}-b_{2,-1}\right) \\
\frac{w t+1}{q_{t+1}}+H & =\gamma_{t} e_{t} \frac{1}{q_{t}}-\gamma_{t} H \forall t \geq 1
\end{array}\right.\right.
$$

or equivalently

$$
\frac{1}{H q_{1}}=\frac{\gamma_{2,0} e_{2,0}}{e_{2,1}} \frac{1}{H q_{0}}-\frac{1}{e_{2,1}}\left(1+\gamma_{2,0} \frac{L+b_{1}^{*}-b_{2,-1}}{L+b_{1}^{*}+b_{2}^{*}}\right), \quad \frac{1}{H q_{t+1}}=\frac{\gamma_{t} e_{t}}{w_{t+1}} \frac{1}{H q_{t}}-\frac{1+\gamma_{t}}{w_{t+1}} \quad \forall t \geq 1
$$

From this, we can compute that, for any $t \geq 2$,

$$
\begin{aligned}
\frac{1}{H q_{t}}= & \frac{\gamma_{t-1} e_{t-1}}{w_{t}}\left(\frac{\gamma_{t-2} e_{t-2}}{w_{t-1}} \frac{1}{H q_{t-2}}-\frac{1+\gamma_{t-2}}{w_{t-1}}\right)-\frac{1+\gamma_{t-1}}{w_{t}} \\
= & \frac{\gamma_{t-1} e_{t-1}}{w_{t}} \frac{\gamma_{t-2} e_{t-2}}{w_{t-1}} \frac{1}{H q_{t-2}}-\frac{\gamma_{t-1} e_{t-1}}{w_{t}} \frac{1+\gamma_{t-2}}{w_{t-1}}-\frac{1+\gamma_{t-1}}{w_{t}} \\
= & \cdots=\frac{\gamma_{t-1} e_{t-1}}{w_{t}} \cdots \frac{\gamma_{1} e_{1}}{w_{2}} \frac{1}{H q_{1}} \\
& \quad-\left(\frac{1+\gamma_{t-1}}{w_{t}}+\frac{\gamma_{t-1} e_{t-1}}{w_{t}} \frac{1+\gamma_{t-2}}{w_{t-1}}+\cdots+\frac{\gamma_{t-1} e_{t-1}}{w_{t}} \cdots \frac{\gamma_{2} e_{2}}{w_{3}} \frac{\left(1+\gamma_{1}\right)}{w_{2}}\right) \\
= & \frac{\gamma_{t-1} e_{t-1}}{w_{t}} \cdots \frac{\gamma_{0} e_{0}}{w_{1}} \frac{1}{H q_{0}} \\
& \quad-\left(\frac{1+\gamma_{t-1}}{w_{t}}+\frac{\gamma_{t-1} e_{t-1}}{w_{t}} \frac{1+\gamma_{t-2}}{w_{t-1}}+\cdots+\frac{\gamma_{t-1} e_{t-1}}{w_{t}} \cdots \frac{\gamma_{1} e_{1}}{w_{2}} \frac{1+\gamma_{2,0} \frac{L+b_{1}^{*}-b_{2,-1}}{L+b_{1}^{*}+b_{2}^{*}}}{w_{1}}\right) \\
= & \frac{1}{H q_{0}} \Gamma_{t}-D_{t} .
\end{aligned}
$$

By consequence, we obtain (22).

Condition $q_{t}>0$ is equivalent to $D_{t} / \Gamma_{t}<1 /\left(H q_{0}\right)$. We see that

$$
\begin{aligned}
\frac{D_{t}}{\Gamma_{t}} & =\frac{\frac{1+\gamma_{t-1}}{w_{t}}+\frac{\gamma_{t-1} e_{t-1}}{w_{t}} \frac{1+\gamma_{t-2}}{w_{t-1}}+\cdots+\frac{\gamma_{t-1} e_{t-1}}{w_{t}} \cdots \frac{\gamma_{1} e_{1}}{w_{2}} \frac{1+\gamma_{2,0} \frac{L+b_{1}^{*}-b_{2,-1}}{L+b_{1}^{*}+b_{2}^{*}}}{w_{1}}}{\frac{\gamma_{t-1} e_{t-1}}{w_{t}} \cdots \frac{\gamma_{0} e_{0}}{w_{1}}} \\
& =\frac{R_{1}^{*} \cdots R_{t-1}^{*}}{e_{t-1}}\left(1+\frac{1}{\gamma_{t-1}}\right)+\cdots+\frac{1}{e_{0}}\left(\frac{1}{\gamma_{0}}+\frac{L+b_{1}^{*}-b_{2,-1}}{L+b_{1}^{*}+b_{2}^{*}}\right)
\end{aligned}
$$


where recall that $\gamma_{0}=\gamma_{2,0}, w_{1} \equiv e_{2,1}$, and $\frac{\gamma_{t-1} e_{t-1}}{w_{t}}=\frac{1}{R_{t}^{*}}$. By combining this with $D_{t} / \Gamma_{t}<1 /\left(H q_{0}\right)$, we get that $\sum_{t=1}^{\infty} \frac{R_{1}^{*} \cdots R_{t}^{*}}{e_{t}}<\infty$.

Part 2. We have to check that (1) prices and consumptions are strictly positive, and (2) all conditions in Lemma 4 are satisfied.

Since $e_{t}-w_{t}>0$, condition $q_{0}<\frac{\Gamma_{t}}{H\left(D_{t}+\frac{2}{e_{t}-w_{t}}\right)}$ is equivalent to $\frac{1}{H q_{0}} \Gamma_{t}-D_{t}>\frac{2}{e_{t}-w_{t}}$ which implies that $\Gamma_{t}>H q_{0} D_{t}$ and $e_{t}-w_{t}>2 H q_{t}$. We have $q_{t}>0$ because $\Gamma_{t}>H q_{0} D_{t}$. Condition $e_{t}-w_{t} \geq 2 H q_{t} \forall t$ ensures that consumptions given by (9b-9c) are strictly positive.

Our construction $\frac{1}{H q_{t}}=\frac{1}{H q_{0}} \Gamma_{t}-D_{t} \forall t \geq 1$ ensures FOCs (B.5a-B.5c).

By using condition $e_{t}-w_{t} \geq 2 H q_{t}$, we obtain (B.4a) and (B.4b). Moreover, condition $q_{0}<\frac{e_{2,0}-e_{1,0}}{L+2 b_{1}^{*}+b_{1,-1}-b_{2,-1}}$ implies condition (B.4c).

Let us look at $\bar{q}$. We can compute that

$$
\begin{aligned}
& \frac{H D_{t}}{\Gamma_{t}}+\frac{2 H}{\left(e_{t}-w_{t}\right) \Gamma_{t}}=H\left(\frac{R_{1}^{*} \cdots R_{t-1}^{*}}{e_{t-1}}\left(\frac{1}{\gamma_{t-1}}+1\right)+\cdots+\frac{1}{e_{0}}\left(\frac{1}{\gamma_{0}}+\frac{L+b_{1}^{*}-b_{2,-1}}{L+b_{1}^{*}+b_{2}^{*}}\right)\right) \\
& \quad+\frac{2 H}{\left(e_{t}-w_{t}\right) \frac{\gamma_{t-1} e_{t-1}}{w_{t}} \cdots \frac{\gamma_{0} e_{0}}{w_{1}}} \\
& =\frac{H w_{1} \cdots w_{t-1}}{e_{0} \cdots e_{t-1}} \frac{1+\gamma_{t-1}}{\gamma_{0} \cdots \gamma_{t-1}}+\frac{H w_{1} \cdots w_{t-2}}{e_{0} \cdots e_{t-2}} \frac{1+\gamma_{t-2}}{\gamma_{0} \cdots \gamma_{t-2}}+ \\
& \quad+\cdots+\frac{H}{e_{0}}\left(\frac{1}{\gamma_{0}}+\frac{L+b_{1}^{*}-b_{2,-1}}{L+b_{1}^{*}+b_{2}^{*}}\right)+\frac{2 H w_{1} \cdots w_{t}}{e_{0} \cdots e_{t-1}\left(e_{t}-w_{t}\right)} \frac{1}{\gamma_{0} \cdots \gamma_{t-1}}
\end{aligned}
$$

Recall that $H \equiv L+b_{1}^{*}+b_{2}^{*}$. As a result, $\frac{H}{\Gamma_{t}}\left(D_{t}+\frac{2}{e_{t}-w_{t}}\right)$ is increasing in $L, b_{1}^{*}, b_{2}^{*}, w_{t}$ and decreasing in $e_{t}, \gamma_{t}, b_{2,-1}$. By consequence, $\bar{q}$ is decreasing in $L, b_{1}^{*}, b_{2}^{*}, w_{t}, b_{1,-1}$ and increasing in $e_{t}, \gamma_{t}, b_{2,-1}$.

\section{B.2 Proofs for Section 4.3.2}

Proof of Proposition 7. We will prove, by induction, condition (33), i.e.,

$$
\frac{\sigma_{s} a_{s} d_{s}}{1+d_{s} H_{s}}<q_{s-1}<\frac{\alpha_{s} a_{s}}{H_{s}} \forall s \geq 1
$$

This is satisfied for $t=1$ because we choose $q_{0} \in\left(\frac{\sigma_{1} a_{1} d_{1}}{1+d_{1} H_{1}}, \frac{\alpha_{1} a_{1}}{H_{1}}\right)$. Assume that it holds for $s=t$. Let us prove it for $s=t+1$. According to (30) and $q_{t-1}<\frac{\alpha_{t} a_{t}}{H_{t}}$, we have

$$
\begin{aligned}
q_{t}=\frac{\left(1+d_{t} H_{t}\right) q_{t-1}-a_{t} d_{t}}{a_{t}-H_{t} q_{t-1}} & <\frac{\left(1+d_{t} H_{t}\right) \frac{\alpha_{t} a_{t}}{H_{t}}-a_{t} d_{t}}{a_{t}-H_{t} \frac{\alpha_{t} a_{t}}{H_{t}}}=\frac{\frac{\alpha_{t}}{H_{t}}-\left(1-\alpha_{t}\right) d_{t}}{1-\alpha_{t}} \\
& <\frac{\alpha_{t}}{\left(1-\alpha_{t}\right) H_{t}}<\frac{\alpha_{t+1} a_{t+1}}{H_{t+1}}
\end{aligned}
$$


where the last inequality is from $(32 \mathrm{~b})$.

The system (30) and condition $\frac{\sigma_{t} a_{t} d_{t}}{1+d_{t} H_{t}}<q_{t-1}$ imply that

$$
q_{t}=\frac{\left(1+d_{t} H_{t}\right) q_{t-1}-a_{t} d_{t}}{a_{t}-H_{t} q_{t-1}}>\frac{\left(1+d_{t} H_{t}\right) \frac{\sigma_{t} a_{t} d_{t}}{1+d_{t} H_{t}}-a_{t} d_{t}}{a_{t}-H_{t} \frac{\sigma_{t} a_{t} d_{t}}{1+d_{t} H_{t}}}=\frac{\left(\sigma_{t}-1\right) d_{t}}{1-\frac{\sigma_{t} d_{t} H_{t}}{1+d_{t} H_{t}}} .
$$

According to condition $1-\left(\sigma_{t}-1\right) d_{t} H_{t}>$, we have $1-\frac{\sigma_{t} d_{t} H_{t}}{1+d_{t} H_{t}}>0$ which in turn implies that

$$
q_{t}>\left(\sigma_{t}-1\right) d_{t}>\sigma_{t+1} a_{t+1} d_{t+1}
$$

where the last inequality is from the first condition in (32c). Finally, we get that $q_{t}>\frac{\sigma_{t+1} a_{t+1} d_{t+1}}{1+H_{t+1} d_{t+1}}$. Therefore, we have just proved (33).

To prove that $\left(q_{t}\right)$ is a price sequence of an equilibrium, we check that all conditions in Lemma 4 are satisfied. First, since $0<\alpha_{t}<1<\sigma_{t}$, condition (33) ensures that $q_{t}>0 \forall t \geq 0$.

Second, observe that condition (33) implies that $q_{t}<\frac{a_{t+1}}{H_{t+1}}$ and hence $q_{t}<\bar{q}_{t}$. By definition of $\bar{q}_{t}$, the inequalities (B.4a-B.4c) can be rewritten as $q_{t} \leq \bar{q}_{t} \forall t$. So, conditions (B.4a-B.4c) are satisfied. It also ensures that consumptions are strictly positive.

Last, FOCs (B.5a-B.5c) are ensured by the system (30).

Proof of Proposition 8. Part 1. According to Remark 3, there is no bubble if $a<1$. Now, consider the case $a>1$. Suppose that there is a bubble. We must have $\sum_{t} d_{t}<\infty$. There are only two cases.

Case 1. If there is $t_{0}$ such that $q_{t_{0}} \leq \frac{a-1}{h}$, then we have

$$
q_{t_{0}+1}-q_{t_{0}}=\frac{q_{t_{0}}\left(h q_{t_{0}}-(a-1)\right)}{a-h q_{t_{0}}}-d_{t}<0
$$

By induction, we have that $q_{t}<q_{t-1}<(a-1) / h \forall t>t_{0}$. By consequence, the sequence $q_{t}$ decreasingly converges to a value $q \geq 0$. Observe that

$$
\left(q_{t}+d_{t}\right)\left(a-h q_{t-1}\right)=q_{t-1}, \text { and hence } q(a-h q)=q,
$$

So, either $q=0$ or $q=(a-1) / h$. Since $q_{t}<q_{t-1}<(a-1) / h \forall t>t_{0}$, the value $q$ must be strictly lower than $(a-1) / h$. As a result, $q_{t}$ converges to zero.

Case 2. $q_{t}>\frac{a-1}{h} \forall t \geq 0$. Observe that $\left(q_{t}+d_{0}+\cdots+d_{t}\right)-\left(q_{t-1}+d_{0}+\cdots+d_{t-1}\right)=q_{t}+d_{t}-q_{t-1}=\frac{q_{t-1}\left(h q_{t-1}-(a-1)\right)}{a-h q_{t-1}}>0$.

So the sequence $\left(q_{t}+d_{0}+\cdots+d_{t}\right)$ is strictly increasing. Since $\sum_{t} d_{t}<\infty$ and $q_{t}<\frac{a}{h}$, this sequence is bounded, and hence converges. As a result, the sequence $\left(q_{t}\right)$ converges. So, it must converge to $\frac{a-1}{h}$. 
Part 2. We now prove that there is almost one equilibrium satisfying $q_{t}>\frac{a-1}{h} \forall t$, in which asset holdings are given by (8) and agents' consumptions are given by (9a9c). Let $\left(q_{t}\right)$ and $\left(q_{t}^{\prime}\right)$ be two systems of equilibrium prices. We must have $q_{t}<a / h$ and $q_{t}^{\prime}<a / h$.

Define $x_{t}=q_{t}-\frac{a-1}{h}, x_{t}^{\prime}=q_{t}^{\prime}-\frac{a-1}{h}$, then we have $0<x_{t}, x_{t}^{\prime}<1 / h$ and

$$
q_{t}+d_{t}=\frac{q_{t-1}}{a-h q_{t-1}} \Leftrightarrow x_{t}+\frac{a-1}{h}+d_{t}=\frac{x_{t-1}+\frac{a-1}{h}}{a-h\left(x_{t-1}+\frac{a-1}{h}\right)} \Leftrightarrow x_{t}+d_{t}=\frac{a x_{t-1}}{1-h x_{t-1}}
$$

Similarly, we have $x_{t}^{\prime}+d_{t}=\frac{a x_{t-1}^{\prime}}{1-h x_{t-1}^{\prime}}$. Therefore, we get that

$$
x_{t}-x_{t}^{\prime}=\frac{a\left(x_{t-1}-x_{t-1}^{\prime}\right)}{\left(1-h x_{t-1}\right)\left(1-h x_{t-1}^{\prime}\right)} \forall t \geq 1 \text {. }
$$

We will prove that $x_{0}=x_{0}^{\prime}$ (which implies that $\left.q_{t}=q_{t}^{\prime} \forall t\right)$. Without loss of generality, suppose that $x_{0}>x_{0}^{\prime}$. According to (B.23), we have $x_{t}-x_{t}^{\prime}>a\left(x_{t-1}-x_{t-1}^{\prime}\right) \forall t \geq 1$. Therefore, we have $x_{t}-x_{t}^{\prime}>a^{t}\left(x_{0}-x_{0}^{\prime}\right) \forall t \geq 1$. Since $a>1, a^{t}\left(x_{0}-x_{0}^{\prime}\right)$ converges to infinity. So, $x_{t}-x_{t}^{\prime}$ also converges to infinity. However, this cannot happen because both $x_{t}$ and $x_{t}^{\prime}$ belong the interval $(0,1 / h)$.

Proof of Example 2. First, we see that

$$
\begin{aligned}
a_{2 t} & =a_{2 t+1}=a \equiv \frac{\beta e}{w}, \quad H_{2 t}=H_{2 t+1}=h \equiv \frac{H(\beta+1)}{w} \\
2 H \bar{q}_{2 t-1} & \equiv e-w-H d_{2 t-1},
\end{aligned}
$$

So, condition $\frac{a_{t+1}}{H_{t+1}}<\bar{q}_{t} \forall t$ becomes

$$
\begin{aligned}
\frac{\beta\left(e_{2,0}+d_{0} b_{2,-1}\right)}{\beta\left(L+b_{1}^{*}-b_{2,-1}\right)+H} & <\frac{e_{2,0}-e_{1,0}-d_{0}\left(b_{1,-1}-b_{2,-1}\right)}{L+2 b_{1}^{*}+b_{1,-1}-b_{2,-1}} \\
\frac{2 \beta e}{1+\beta} & <e-w-H d_{2 t-1}, \quad \frac{2 \beta e}{1+\beta}<e-w-H d_{2 t} .
\end{aligned}
$$

These conditions and condition $\bar{q}_{t}>0$ are satisfied because we assume that $d_{t}<$ $\frac{\frac{1-\beta}{1+\beta} e-w}{H}$.

Second, observe that condition $\frac{\sigma a d_{1}}{1+d_{1} \frac{H(\beta+1)}{w}}<\frac{\beta e-w}{H(\beta+1)}$ ensures that $\frac{\sigma a d_{1}}{1+d_{1} h}<\frac{a-1}{h}$. So, the interval $\left(\frac{\sigma a d_{1}}{1+d_{1} h}, \frac{a-1}{h}\right]$ is well defined.

We next prove that $q_{s} \in\left(\frac{\sigma a d_{s}}{1+d_{s} h}, \frac{a-1}{h}\right] \forall s \geq 0$. This holds for $s=0$ because $q_{0} \in\left(\frac{\sigma a d_{1}}{1+d_{1} h}, \frac{a-1}{h}\right]$. Assume that it holds for $t-1$, we will prove this for $t$. Indeed, condition $d_{t}<\frac{w}{(\sigma-1)(\beta+1) H}$ is equivalent to $1-\frac{\sigma d_{t} h}{1+d_{t} h}>0$. By combining this with 
$\frac{\sigma-1}{\sigma} \frac{d_{t}}{d_{t+1}}>\frac{\beta e}{w}$, we get that

$$
\begin{aligned}
q_{t} & =\frac{\left(1+d_{t} h\right) q_{t-1}-a d_{t}}{a-h q_{t-1}}>\frac{\left(1+d_{t} h\right) \frac{\sigma a d_{t}}{1+d_{t} h}-a d_{t}}{a_{t}-h \frac{\sigma a d_{t}}{1+d_{t} h}}=\frac{(\sigma-1) d_{t}}{1-\frac{\sigma d_{t} h}{1+d_{t} h}} \\
& >(\sigma-1) d_{t}>\sigma a_{t+1} d_{t+1}>\frac{\sigma a_{t+1} d_{t+1}}{1+H_{t+1} d_{t+1}}
\end{aligned}
$$

We also have

$$
q_{t}-q_{t-1}=\frac{q_{t-1}\left(h q_{t-1}-(a-1)\right)}{a-h q_{t-1}}-d_{t}<0
$$

because $h q_{t-1}<a-1$. So, we have $q_{t}<q_{t-1}<(a-1) / h \forall t$. This in turn implies that $q_{t}<(a-1) / h \forall t$. By consequence, the sequence $q_{t}$ decreasingly converges, and hence it cannot converge to $(a-1) / h$. As a result, it converges to zero.

It remains to prove that $\left(q_{t}\right)$ is a price sequence of an equilibrium. To do so, we verify that all conditions in Lemma 4 are satisfied. First, it is easy to see that $q_{t}>0$ $\forall t \geq 0$. Second, according to $\frac{a_{t+1}}{H_{t+1}}<\bar{q}_{t}$, we have $q_{t}<\frac{a_{t+1}}{H_{t+1}}<\bar{q}_{t}$. This shows that conditions (B.4a-B.4c) are satisfied. It also ensures that consumptions are strictly positive. Last, FOCs (B.5a-B.5c) are ensured by the system (30).

Proof of Example 3. We see that $1-(a-1) x>0$ and $h x d_{0}<1$. So, we can check that $0<h x d_{t}<1$ and $x d_{t}+d_{t}=\frac{a x d_{t-1}}{1-h x d_{t-1}} \forall t \geq 0$. According to the proof of Proposition 8 , the sequence $\left(q_{t}\right)$, defined by $q_{t}=\frac{a-1}{h}+x d_{t} \forall t$, satisfies: $q_{t} \in\left(\frac{a-1}{h}, \frac{a}{h}\right)$ and $q_{t}+d_{t}=\frac{q_{t-1}}{a-h q_{t-1}} \forall t$. In order to prove that $\left(q_{t}\right)$ is a system of prices of an equilibrium at which asset holdings are given by (8) and agents' consumptions are given by (9a-9c), we verify all conditions in Lemma 4 .

As in the proof of Example 2, condition $d_{0}<\frac{\frac{1-\beta}{1+\beta} e-w}{H}$ ensures that $a / h<\bar{q}_{t} \forall t$. Thus, $q_{t}<a / h<\bar{q}_{t} \forall t$. This shows that conditions (B.4a-B.4c) are satisfied. It also ensures that consumptions are strictly positive. Last, FOCs (B.5a-B.5c) are ensured by the system $q_{t}+d_{t}=\frac{q_{t-1}}{a-h q_{t-1}} \forall t$.

\section{B.3 Proofs for Section 4.4}

Proof of Proposition 9. We need an intermediate step.

Lemma 5. Assume that $u_{i}$ is strictly concave and $u_{i}^{\prime \prime}<0$. Then, $\beta_{1,2 t-1} u_{1}\left(c_{1,2 t-1}\right)+$ $\beta_{1,2 t} u_{1}\left(c_{1,2 t}\right)$ and $\beta_{2,2 t} u_{2}\left(c_{2,2 t}\right)+\beta_{2,2 t+1} u_{2}\left(c_{2,2 t+1}\right)$ are strictly positive and strictly increasing in $q_{0}$. 
Proof of Lemma 5. The FOCs in Proposition 1 imply that

$$
\left\{\begin{array}{c}
\gamma_{1,2 t-1} \frac{u_{1}^{\prime}\left(e_{1,2 t}+d_{2 t}\left(L+b_{2}^{*}\right)+q_{2 t} H\right)}{u_{1}^{\prime}\left(e_{1,2 t-1}-b_{1}^{*} d_{2 t-1}-q_{2 t-1} H\right)} \geq \gamma_{2,2 t-1} \frac{u_{2}^{\prime}\left(e_{2,2 t}-d_{2 t} b_{2}^{*}-q_{2 t} H\right)}{u_{2}^{\prime}\left(e_{2,2 t-1}+d_{2 t-1}\left(L+b_{1}^{*}\right)+q_{2 t-1} H\right)} \\
\gamma_{2,2 t} \frac{u_{2}^{\prime}\left(e_{2,2 t+1}+d_{2 t+1}\left(L+b_{1}^{*}\right)+q_{2 t+1} H\right)}{u_{2}^{\prime}\left(e_{2,2 t}-d_{2 t} b_{2}^{*}-q_{2 t} H\right)} \geq \gamma_{1,2 t} \frac{u_{1}^{\prime}\left(e_{1,2 t+1}-b_{1}^{*} d_{2 t+1}-q_{2 t+1} H\right)}{u_{1}^{\prime}\left(e_{1,2 t}+d_{2 t}\left(L+b_{2}^{*}\right)+q_{2 t} H\right)}
\end{array}\right.
$$

and

$$
\begin{array}{r}
\beta_{1,2 t-1} q_{2 t-1} u_{1}^{\prime}\left(e_{1,2 t-1}-b_{1}^{*} d_{2 t-1}-q_{2 t-1} H\right)=\beta_{1,2 t} u_{1}^{\prime}\left(e_{1,2 t}+d_{2 t}\left(L+b_{2}^{*}\right)+q_{2 t} H\right)\left(q_{2 t}+d_{2 t}\right) \\
\beta_{2,2 t} q_{2 t} u_{2}^{\prime}\left(e_{2,2 t}-b_{2}^{*} d_{2 t}-q_{2 t} H\right)=\beta_{2,2 t+1}\left(q_{2 t+1}+d_{2 t+1}\right) u_{2}^{\prime}\left(e_{2,2 t+1}+d_{2 t+1}\left(L+b_{1}^{*}\right)+q_{2 t+1} H\right)
\end{array}
$$

Taking the derivatives of both sides of equalities with respect to $q_{0}$ and combining with FOCs, we have

$$
\begin{gathered}
\beta_{1,2 t-1} q_{2 t-1}^{\prime}\left(q_{0}\right) u_{1}^{\prime}\left(c_{1,2 t-1}\right) \leq \beta_{1,2 t-1} q_{2 t-1}^{\prime}\left(q_{0}\right)\left(u_{1}^{\prime}\left(c_{1,2 t-1}\right)-q_{2 t-1} H u_{1}^{\prime \prime}\left(c_{1,2 t-1}\right)\right) \\
=\beta_{1,2 t} q_{2 t}^{\prime}\left(q_{0}\right)\left(u_{1}^{\prime}\left(c_{1,2 t}\right)+H u_{1}^{\prime \prime}\left(c_{1,2 t}\right)\left(q_{2 t}+d_{2 t}\right)\right) \\
<\beta_{1,2 t} q_{2 t}^{\prime}\left(q_{0}\right) u_{1}^{\prime}\left(c_{1,2 t}\right) \\
\beta_{2,2 t} q_{2 t}^{\prime}\left(q_{0}\right) u_{2}^{\prime}\left(c_{2,2 t}\right) \leq \beta_{2,2 t} q_{2 t}^{\prime}\left(q_{0}\right)\left(u_{2}^{\prime}\left(c_{2,2 t}\right)-q_{2 t} H u_{2}^{\prime \prime}\left(c_{2,2 t}\right)\right) \\
=\beta_{2,2 t+1} q_{2 t+1}^{\prime}\left(q_{0}\right)\left(u_{2}^{\prime}\left(c_{1,2 t+1}\right)+H u_{2}^{\prime \prime}\left(c_{2,2 t+1}\right)\left(q_{2 t+1}+d_{2 t+1}\right)\right) \\
<\beta_{2,2 t+1} q_{2 t+1}^{\prime}\left(q_{0}\right) u_{2}^{\prime}\left(c_{2,2 t+1}\right) .
\end{gathered}
$$

where we also use $u_{i}^{\prime \prime}<0$ (the function $u_{i}$ is strictly concave) for $i=1,2$.

By consequence, we have that

$$
\begin{aligned}
& \frac{\partial}{\partial q_{0}}\left(\beta_{1,2 t-1} u_{1}\left(c_{1,2 t-1}\right)+\beta_{1,2 t} u_{1}\left(c_{1,2 t}\right)\right) \\
& =-H \beta_{1,2 t-1} u_{a}^{\prime}\left(c_{1,2 t-1}\right) q_{2 t-1}^{\prime}\left(q_{0}\right)+H \beta_{1,2 t} u_{a}^{\prime}\left(c_{1,2 t}\right) q_{2 t}^{\prime}\left(q_{0}\right)>0
\end{aligned}
$$

and

$$
\begin{aligned}
& \frac{\partial}{\partial q_{0}}\left(\beta_{2,2 t} u_{2}\left(c_{2,2 t}\right)+\beta_{2,2 t+1} u_{2}\left(c_{2,2 t+1}\right)\right) \\
& =-H \beta_{2,2 t} u_{b}^{\prime}\left(c_{2,2 t}\right) q_{2 t}^{\prime}\left(q_{0}\right)+H \beta_{2,2 t+1} u_{b}^{\prime}\left(c_{2,2 t+1}\right) q_{2 t+1}^{\prime}\left(q_{0}\right)>0 .
\end{aligned}
$$

We now prove Proposition 9. According to Lemma 5 , the sequences $\beta_{1,2 t-1} u_{1}\left(c_{1,2 t-1}\right)+$ $\beta_{1,2 t} u_{1}\left(c_{1,2 t}\right)$ and $\beta_{2,2 t} u_{2}\left(c_{2,2 t}\right)+\beta_{2,2 t+1} u_{2}\left(c_{2,2 t+1}\right)$ are strictly positive and strictly increasing in $q_{0}$.

We now prove that the function $W_{i}(q)$ is increasing in $q$. Notice that we cannot directly prove this by looking at $\sum_{t \geq 0} \frac{\partial}{\partial q_{0}}\left(\beta_{i, t} u_{i}\left(c_{i, t}\right)\right)$ for $i=1,2$, because it is unclear 
that this series converge. So, we will prove our result as follows. Let $q_{0}$ and $q_{0}^{\prime}$ be the two initial prices of two equilibria $\left(q_{t},\left(c_{i, t}\right)_{i}\right)$ and $\left(q_{t}^{\prime},\left(c_{i, t}^{\prime}\right)_{i}\right)$. Assume that $q_{0}>q_{0}^{\prime}$. Consider agent 1 and denote $A_{T} \equiv \sum_{t=0}^{T} \beta_{1, t}\left(u_{1}\left(c_{1, t}\right)-u_{1}\left(c_{1, t}^{\prime}\right)\right)$. We will prove that $A_{T}$ converges to a strictly positive number when $T$ tends to infinity. Indeed, we see that $A_{2 T}=\sum_{t=0}^{2 T} \beta_{1, t}\left(u_{1}\left(c_{1, t}\right)-u_{1}\left(c_{1, t}^{\prime}\right)\right)$ is strictly positive and increasing in $T$ (because $\beta_{1,2 t-1} u_{1}\left(c_{1,2 t-1}\right)+\beta_{1,2 t} u_{1}\left(c_{1,2 t}\right)$ is strictly increasing in $q_{0}$ et $c_{1,0}$ is increasing in $\left.q_{0}\right)$. So, it converges to a strictly positive value.

We now observe that

$$
A_{2 T+1}=\sum_{t=0}^{2 T} \beta_{1, t}\left(u_{1}\left(c_{1, t}\right)-u_{1}\left(c_{1, t}^{\prime}\right)\right)+\beta_{1,2 T+1}\left(u_{1}\left(c_{1,2 T+1}\right)-u_{1}\left(c_{1,2 T+1}^{\prime}\right)\right)
$$

It is easy to see that $\beta_{1,2 T+1}\left(u_{1}\left(c_{1,2 T+1}\right)-u_{1}\left(c_{1,2 T+1}^{\prime}\right)\right)$ converges to zero because both $\beta_{1,2 T+1} u_{1}\left(c_{1,2 T+1}\right)$ and $\beta_{1,2 T+1} u_{1}\left(c_{1,2 T+1}^{\prime}\right)$ converge to zero. By consequence, we have $A_{2 T}$ and $A_{2 T+1}$ converge to the same value. So, $A_{t}$ converges to a strictly positive value when $t$ tends to infinity.

By using the same method, we can prove that $\sum_{t=0}^{T} \beta_{2, t}\left(u_{2}\left(c_{2, t}\right)-u_{2}\left(c_{2, t}^{\prime}\right)\right)$ converges to a strictly positive number when $T$ tends to infinity.

\section{References}

Araujo, A., Novinski, R., Pascoa, M.R., 2011. General equilibrium, wariness and efficient bubbles. Journal of Economic Theory 46, p. 785-811.

Araujo, A., Pascoa, M.R., Torres-Martinez, J.P., 2011. Long-lived collateralized assets and bubbles. Journal of Mathematical Economics 47, 260-271.

Alvarez, F., Jermann, U.J., 2000. Efficiency, equilibrium, and asset pricing with risk of default. Econometric 68(4), p. 775-797.

Becker, R., Bosi, S., Le Van, C., Seegmuller, T., 2015. On existence and bubbles of Ramsey equilibrium with borrowing constraints. Economic Theory 58, 329-353.

Benhabib, J., Farmer R.E.A., 1999. Indeterminacy and sunspots in macroeconomics. In: Taylor, J.B., Woodford, M., (Eds.) Handbook of Macroeconomics, vol. 1, issue: part A, p. 387-448, Elsevier Science BV.

Barbie, M., Hillebrand, M., 2018. Bubbly Markov equilibria, Economic Theory, vol. 66, p. 627-679.

Bewley T., 1980. The optimal quantity of money. In: Kareken, J.H., Wallace, N., (Eds.) Models of Monetary Economics, Federal Reserve Bank of Minneapolis, p. 169-210. 
Bloise G, Citanna A., 2019. Asset shortages, liquidity and speculative bubbles, Journal of Economic Theory, vol. 183, p. 952-990.

Bosi, S., Ha-Huy, T., Le Van, C., Pham, C.T., Pham, N.-S., 2018a. Financial bubbles and capital accumulation in altruistic economies, Journal of Mathematical Economics, vol. 75 , p. $125-139$.

Bosi, S., Le Van, C., Pham, N.-S., 2017a. Asset bubbles and efficiency in a generalized two-sector model, Mathematical Social Sciences, vol. 88, p. 37-48.

Bosi, S., Le Van, C., Pham, N.-S., 2017b. Intertemporal equilibrium with heterogeneous agents, endogenous dividends and collateral constraints, CES working paper series, No. 2015.67R.

Bosi, S., Le Van, C., Pham, N.-S., 2018b. Intertemporal equilibrium with heterogeneous agents, endogenous dividends and collateral constraints, Journal of Mathematical Economics, vol. 76 , p. 1-20.

Bosi, S., Le Van, C., Pham, N.-S., 2019. Real indeterminacy and dynamics of asset price bubbles in general equilibrium, MPRA Paper, No. MPRA_paper_96834.

Brunnermeier, M.K., Oehmke, M., 2012. Bubbles, financial crises, and systemic risk. Handbook of the Economics of Finance, vol. 2.

Doblas-Madrid A., 2012. A robust model of bubbles with multidimensional uncertainty, Econometrica 80, p. 1845-1893.

Farhi, E., Tirole, J., 2012. Bubbly liquidity. Review of Economic Studies 79, p. 678-706.

Farmer R.E.A., 2019. The Indeterminacy Agenda in Macroeconomics, NBER Working Paper, No. 25879.

Gali, J., 2014. Monetary Policy and Rational Asset Price Bubbles. American Economic Review 104, p. 721-752.

Gali, J., 2018. Monetary Policy and Bubbles in a New Keynesian Model with Overlapping Generations. Economics Working Papers 1561, Department of Economics and Business, Universitat Pompeu Fabra.

Huang, K. X. D., Werner, J., 2000. Asset price bubbles in Arrow-Debreu and sequential equilibrium. Economic Theory 15, p. 253-278.

Hirano, T., Yanagawa, N., 2017. Asset Bubbles, Endogenous Growth, and Financial Frictions. The Review of Economic Studies, vol. 84, p. 406-443.

Kamihigashi, T., 2002. A simple proof of the necessity of the transversality condition. Economic Theory 20, 427-433.

Kehoe, T.J., Levine, D.K., 1985. Comparative statics and perfect foresight in infinite horizon economies. Econometrica 53, No. 2, p. 433-453. 
Kehoe, T.J., Levine, D.K., Romer, P.M., 1990. Determinacy of equilibria in dynamic models with finitely many consumers. Journal of Economic Theory 50, p. 1-21.

Kiyotaki, N., Moore, J., 1997. Credit cycles. Journal of Political Economy, 105(2), p. 211248.

Kocherlakota, N. R., 1992. Bubbles and constraints on debt accumulation. Journal of Economic Theory 57, p. 245 - 256.

Kocherlakota, N. R., 2008. Injecting rational bubbles. Journal of Economic Theory 142, p. $218-232$.

Le Van, C., Pham, N.-S., 2016. Intertemporal equilibrium with financial asset and physical capital. Economic Theory 62, p. 155-199.

Levine, D. K., Zame, W. R., 1996. Debt constraints and equilibrium in infinite horizon economies with incomplete markets. Journal of Mathematical Economics 26, p. 103-131.

Ljungqvist, L., Sargent, T. J. 2012. Recursive Macroeconomic Theory, third edition, The MIT Press

Lucas, R. E., 1978. Asset prices in an exchange economy. Econometrica 46, p. 1429-1445.

Magill, M., Quinzii, M., 1994. Infinite Horizon Incomplete Markets. Econometrica 62, p. 853-880.

Magill, M., Quinzii, M., 1996. Incomplete markets over an infinite horizon: Long-lived securities and speculative bubbles. Journal of Mathematical Economics 26, p. 133-170.

Martin, A., Ventura, J., 2012. Economic growth with bubbles. American Economic Review 102, p. $3033-3058$.

Martin, A., Ventura, J., 2018. The Macroeconomics of Rational Bubbles: A User's Guide. Annual Review of Economics, 10(1), 505-539. https://doi.org/10.1146/annureveconomics-080217-053534

Miao, J., 2014. Introduction to economic theory of bubbles. Journal of Mathematical Economics 53, p. 130-136.

Miao, J., Wang, P., 2012. Bubbles and total factor productivity. American Economic Review: Papers and Proceedings 102, p. 82-87.

Miao, J., Wang, P., 2018. Asset bubbles and credit constraints. American Economic Review 108, p. 2590-2628.

Montrucchio, L., 2004. Cass transversality condition and sequential asset bubbles. Economic Theory 24, p. 645-663.

Pascoa, M.R., Petrassi, M., Torres-Martinez, J.P., 2011. Fiat money and the value of binding portfolio constraints. Economic Theory 46, p. 189-209. 
Santos, M. S., Woodford, M., 1997. Rational asset pricing bubbles. Econometrica 65, p. $19-57$.

Scheinkman J. A., Weiss L., 1986. Borrowing constraints and aggregate economic activity. Econometrica 54 (1), p. 23-45.

Tirole, J., 1982. On the possibility of speculation under rational expectations. Econometrica 50, p. $1163-1181$.

Tirole, J., 1985. Asset bubbles and overlapping generations. Econometrica 53, p. 1499-1528.

Townsend, R., 1980. Models of Money with Spatially Separated Agents. In: Kareken, J.H., Wallace, N., (Eds.) Models of Monetary Economics, Federal Reserve Bank of Minneapolis, p. 265-303.

Werner, J., 2014. Rational asset pricing bubbles and debt constraints. Journal of Mathematical Economics, vol. 53, p. 145-152. 